\section{Inversión pública y plusvalías urbanas \\ Análisis espacial y efectos no lineales de la proximidad de la red de subterráneos sobre los precios de la vivienda en Buenos Aires}

(4) Sergio Andrés Rosanovich

CONICET / Instituto de Altos Estudios Sociales, Universidad Nacional de San Martín, Argentina.

\author{
Ana Paula Di Giovambattista \\ CONICET / Centro de Innovación de los Trabajadores, Universidad Metropolitana para la \\ Educación y el Trabajo, Argentina.
}

Recibido: 20 de marzo de 2018. Aceptado: 4 de julio de 2018.

\title{
Resumen
}

En relación a la discusión sobre la captación y recuperación de plusvalías urbanas, uno de los insumos necesarios para diseñar una política económica es la identificación no solo de la dimensión del efecto sino también del universo de unidades afectadas a partir del desarrollo urbano. En este trabajo se realiza tal análisis sobre los inmuebles ofertados para la venta en la Ciudad de Buenos Aires en relación a su localización respecto a la red de subterráneos. Asimismo, se implementa por primera vez un modelo no lineal de precios hedónicos con dependencia espacial a fin de captar la incidencia sobre este mercado mediante el uso de una base de datos propia de precios de oferta. Se constata la presencia de un efecto no lineal del precio por metro cuadrado en función de la distancia a las estaciones de subte, negativo para el rango de 0 a 200 metros (-5\%) y positivo para el de 300 a 600 metros $(+3,6 \%)$. Adicionalmente, se presenta un detalle para 2017-2018 del estado actual de las heterogeneidades entre líneas de subte, dando cuenta de diferencias en la distribución espacial de los precios de las propiedades, parcialmente determinada por los atributos (v.g. vigilancia, pileta, gimnasio) que poseen los edificios en los distintos barrios de la ciudad.

\section{Public investment and urban capital gains. Spatial analysis and non-linear effects between the proximity to the subway network and house prices in Buenos Aires}

\footnotetext{
Abstract

As regards the capture and recovery of urban capital gains, one of the requirements for the design of public policy is the identification not only of the measure of the effect
}

Palabras clave

Mercado inmobiliario Plusvalías urbanas Transporte público Econometría espacial

\section{Keywords}

Real-estate market Urban capital gains Public transport Spatial econometrics 
but also of the universe of affected properties as a result of urban development. In this article, the selling offers of apartments in Buenos Aires are analyzed in relation to its localization and distance to the subway network. Furthermore, for the first time a non-linear hedonic price model with spatial dependence is implemented in order to show the impact over real-estate market using a new dataset of selling prices. The presence of a non-linear effect of the price per square meter based on the distance to the subway stations is verified, negative for the range between 0 and 200 meters $(-5 \%)$ and positive for the range of 300 to 600 meters $(+3,6 \%)$. In addition, a detailed description of the current heterogeneities between subway lines is included, stressing the differences in the spatial distribution of house prices, partially determined by the attributes (v.g. vigilance, swimming pool, gym) of the buildings in the different neighborhoods of the city.

\section{Introducción}

Los procesos de valorización de inmuebles han constituido objeto de estudio de numerosas investigaciones científicas. Entre las disciplinas que han abordado estos fenómenos se destaca la ciencia económica, a partir de la cual se ha estudiado la incidencia de la política de créditos hipotecarios en aumentos de precios de inmuebles y la emergencia de burbujas inmobiliarias como causa de potenciales crisis financieras (Chaney, Sraer, y Thesmar, 2012; Mera, 2016). Asimismo, el rol sobre la distribución del ingreso y el mercado de trabajo debido al efecto riqueza que beneficia a quienes son propietarios y la capacidad de generación de empleo que detenta la actividad de la construcción (Linneman y Wachter, 1989; Rodríguez y Di Virgilio, 2014). De manera complementaria, el urbanismo y la geografía han analizado las transformaciones del ejido urbano derivadas de las conductas especulativas generadas a partir del incremento en el valor de las propiedades (Loaiza Rincón y Morales, 2002; Baer y Kauw, 2016); o el derecho, desde donde se han examinado y discutido las facultades efectivas de los gobiernos para intervenir sobre tales procesos (Mattos, 2006; Fernandes y Maldonado, 2009).

Ahora bien, una dinámica creciente de los valores patrimoniales puede responder a múltiples causas. La primera instancia para su análisis, por tanto, debiera basarse en una correcta identificación en relación al disparador de dicha modificación de los precios de inmuebles. Al respecto, estimamos necesaria una inaugural categorización entre vías de valorización, que excedan a la dinámica macroeconómica, producto de: a) el esfuerzo individual del propietario (e.g. mejoramiento de materiales constitutivos); b) intervenciones directas en el territorio (e.g. inversiones en infraestructura, dotación de equipamiento y servicios urbanos) o indirectas (e.g. de modificación de normativas urbanas, rezonificación del suelo, actualización del Código Urbanístico, autorización de nuevos usos del suelo o modificación de los indicadores de edificabilidad, entre otras) por parte del Estado; y c) el esfuerzo individual de un tercero que pueda generar una externalidad positiva en la zona.

En torno a esa clasificación, cuando los incrementos en el valor de inmuebles responden a intervenciones gubernamentales se configuran escenarios de apropiación individual del efecto riqueza acontecido. Ante tales situaciones, la literatura de economía urbana ha discutido extensamente la diversidad de instrumentos fiscales que promuevan la recuperación de plusvalías urbanas bajo la forma de impuestos o contribuciones específicas (Clichevsky, 2001, 2004; Jaramillo, 2006). Por plusvalías urbanas nos referimos a la valorización inmobiliaria socialmente generada y apropiada individualmente por los propietarios en forma de un aumento del precio de venta de una vivienda. Se profundizará sobre este concepto en la siguiente sección.

\section{Palavras-chave}

mercado imobiliário Ganhos de capital urbano Transporte público Econometria espacial 
Sin embargo, la potencialidad redistributiva de la política fiscal requiere a priori un adecuado examen de mensura de tales valorizaciones. En la presente contribución se hará referencia particularmente a aquella acontecida como resultado de un tipo de intervención Estatal directa sobre el territorio: la dotación de infraestructura de transporte subterráneo para la Ciudad Autónoma de Buenos Aires (CABA). En consecuencia, nuestro trabajo se propone: i) reseñar abordajes y análisis cuantitativos previos respecto a la discusión aludida, para el contexto local e internacional; y ii) efectuar una medición del impacto diferencial, considerando efectos no lineales, para el mercado inmobiliario de venta en 2017. Para ello, los modelos de Precios Hedónicos permiten estimar la contribución de cada atributo (de la propia vivienda, del edificio y de la localización) al precio de venta.

En este sentido, la investigación se articula en un encadenamiento de etapas que van desde la identificación del efecto de las políticas urbanas -en particular la inversión pública en transporte- sobre el mercado inmobiliario hasta la acción de la política tributaria con la meta de recuperar las plusvalías urbanas. El presente texto conforma el primer eslabón de tal proceso, y se buscará contrastar la hipótesis de que aun existiendo heterogeneidades entre los precios de las propiedades cercanas a la red de subterráneos para diferentes estaciones y trazas, existe un efecto adicional no lineal asociado al grado de proximidad de las propiedades con respecto a las estaciones en general.

A tal efecto, el artículo se estructura de la siguiente manera: luego de la presente introducción, en la segunda sección se expone el origen y evolución del concepto de renta y su aplicación al campo de la economía urbana. Posteriormente, las definiciones esgrimidas se complementan con una revisión de las investigaciones académicas en torno a la medición del impacto sobre precios de inmuebles que generan las intervenciones gubernamentales en el territorio. Luego se presenta esquemáticamente la red de subterráneos de la Ciudad de Buenos Aires. A continuación, se exponen las fuentes de información y técnicas metodológicas utilizadas para la mensura de la magnitud y sentido de la incidencia de la proximidad a la red de subterráneos sobre los valores de las propiedades, y, subsiguientemente los resultados obtenidos para las diversas líneas de subterráneos. Finalmente, se concluye con el apartado de reflexiones.

\section{Elementos conceptuales}

Con miras a establecer definiciones en torno a las rentas urbanas, resulta necesario realizar una breve revisión de ciertos conceptos vinculados a la tierra rural, dado que constituyen los antecedentes más relevantes para la construcción de nociones y categorías para el estudio aquí planteado.

Desde la teoría económica, la escuela clásica, y en particular Ricardo (1817), ha intentado dar explicación a la formación de los precios de la tierra, en el marco del análisis de la distribución del producto económico entre clases sociales. Así, desde una perspectiva dinámica para la compresión del sistema económico, Ricardo estableció -continuando con las ideas de Smith (1776)- que en una sociedad articulada por la división del trabajo, los salarios se fijan al nivel que posibilita la reproducción de la fuerza de trabajo. Y que, paralelamente, la retribución a capitalistas y terratenientes -utilidades y renta, respectivamente- componen lo que se denomina excedente -entendido como el remanente del producto del proceso productivo luego de cubrir los costos de producción- (Roncaglia, 2006; Teubal, 2012).

En Principios de Economía y Tributación (1817), Ricardo define a la renta como “(...) aquella parte del producto de la tierra que se paga al terrateniente por el uso de las 
energías originarias e indestructibles del suelo" (Ricardo, 1817:51). Tal especificación implica una relación estrecha entre la renta percibida y las condiciones naturales de la tierra, y es a partir de este vínculo que se incorporan al análisis características específicas del mercado de suelo -i.e. irreproductibilidad, escasez e indestructibilidad- para la enunciación de la Teoría de la Renta Diferencial.

En términos de Ricardo, luego de una etapa inicial en la que se pondrán bajo cultivo las parcelas más fértiles, la expansión y progreso de la sociedad abre la necesidad de ampliar la superficie destinada a la producción, implicando la incorporación de tierras de calidad inferior. Es ese diferencial de calidad lo que da lugar a la renta asociada a la tierra más fértil, de modo que para cada parcela la renta estará dada por la diferencia entre los costos unitarios de producción en tierras menos fértiles, y los costos unitarios en la tierra considerada, multiplicada tal discrepancia por la cantidad de producto obtenido de ella. El proceso es iterativo, y a medida que se adicionan nuevas tierras de menor fertilidad, se reduce la productividad marginal -i.e. Ley de Rendimientos Decrecientes ${ }^{1}$ - (Teubal, 2012). Así, se configura un complejo de rentas en función del número de categorías de tierras que se distingan por su nivel de fertilidad y aptitud para el cultivo, tal que la renta de las tierras cultivadas menos fértiles resultará nula.

Dos aspectos detentan relevancia en la generación de renta. El primero se vincula con el efecto de localización de las parcelas, destacado por Von Thünen (1826) en su trabajo seminal sobre ciudad monocéntrica, y retomado por diversos autores (Isard, 1956; Fujita, Krugman y Venables, 1999). De acuerdo a Von Thünen, la renta asociada a una parcela con determinada localización se estima en relación a su distancia al centro -considerando homogeneidad en términos de fertilidad-, y es susceptible de ser medida en función del costo de transporte. Mientras una unidad de superficie de tierra en el centro generará una renta dada por el rendimiento por unidad de producción multiplicada por el ingreso neto (diferencia entre su precio y costo de producción), para toda parcela fuera del centro, a esa renta deberá sustraerse el costo asociado al transporte (tasa de transporte por unidad de distancia multiplicada por la distancia de la parcela al centro) (Dunn, 1954).

Por su parte, Haig (1926) define a la renta urbana o residencial como la carga que el propietario puede imponer a otros agentes como consecuencia del ahorro de costos de transporte que se derivan de la localización de la propiedad en cuestión. En adición al énfasis puesto sobre la característica de localización, la corriente iniciada por Von Thünen puso sobre relieve el hecho de que las categorías inicialmente planteadas para el ámbito del suelo rural presentaban correspondencia en la dinámica de funcionamiento del suelo urbano (Alonso, 1960).

El segundo elemento que se desprende de la definición original de renta ricardiana se liga a la naturaleza misma del suelo como factor de producción. En el sentido de que el proceso productivo incorpora a la tierra como factor productivo per se-que genera un retorno periódico como resultado de su uso-, por un lado, y como espacio sobre el que se generan inversiones de capital -i.e. capitalización del suelo-. Al respecto, el autor establece que:

(...) sólo una parte de esa remuneración o renta se asocia con las energías originarias e indestructibles del suelo; la otra parte se pagaría por el uso del capital empleado para mejorar la calidad de la tierra, y para erigir los edificios que se van necesitando con objeto de obtener y conservar el producto (Ricardo, 1817: 51).

A partir de la extensión y profundización de la disquisición precedente, una línea de investigación iniciada por Jaramillo a fines de la década de 1970 ha intentado elaborar
1. La Ley o Regularidad de Rendimientos Marginales Decrecientes refiere al hecho estilizado de productividad creciente a tasa decreciente que tiene lugar al considerar una cantidad fija de tierra, e ir adicionando sucesivas cantidades de trabajo. El resultado es que inicialmente el producto generado con las incorporaciones marginales será más que proporcional al incremento en el factor productivo, pero, a medida que la cantidad total de trabajo se incrementa, las adiciones marginales generarán aumentos en el producto total de menor magnitud (Ricardo, 1817). 
una teoría de la renta urbana (Jaramillo, 1994; 1977). En efecto, el análisis teórico desde un abordaje marxista hace hincapié en la distinción entre la relación tierra/proceso productivo para el caso rural y el caso urbano.

En el ámbito rural, los bienes agrícolas constituyen el producto a vender, siendo éste móvil en relación al suelo. En contraste, en el ámbito urbano se trata de la propia vivienda, indivisible e inmóvil con respecto al suelo. Es decir, que en la esfera urbana el bien:

\section{(...) se produce en el mismo lugar en que se consume y las características de la tierra son un determinante muy importante para este consumo. El producto en este caso no está ligado a la tierra solamente durante su período de producción, sino que este nexo se extiende al período de circulación y de consumo del bien (Jaramillo, 2003:34).}

De manera que para el caso rural la articulación entre tierra y proceso productivo se define como Articulación Única o Simple, al tiempo que en el caso urbano es Articulación Compleja. Esta Articulación Compleja se conforma, entonces, por la Articulación Primaria (i.e. construcción) y la Articulación Secundaria, que refiere al conjunto de actividades urbanas que requieren del espacio construido sobre el suelo (Jaramillo, 1977).

Dada la complejidad inherente al vínculo entre suelo urbano y el proceso productivo, las rentas urbanas pueden incrementarse como consecuencia del crecimiento poblacional -que impulsa la anexión de nuevos territorios desde el ámbito rural al periurbano y urbano-, pero, adicionalmente, pueden ampliarse como respuesta a la incorporación de mejoras o inversiones. Y, además, ser categorizadas en rentas primarias y rentas secundarias, siendo las últimas aquellas que se desprenden del hecho de que los consumidores pueden estar dispuestos a pagar un adicional por el espacio construido en una cierta localización (Topalov y Enrique, 1984; Jaramillo, 2003). ${ }^{2}$ Emerge, por consiguiente, una decisiva influencia de la infraestructura urbana sobre los valores de los inmuebles, siendo la provisión de servicios urbanos un mecanismo de potencial generación de este tipo de rentas (Morales Schechinger, 2005).

En efecto, las plusvalías urbanas hacen referencia a las valorizaciones inmobiliarias socialmente generadas (Munévar y Hernández, 2017). De allí que, ante la existencia de escenarios de apropiación individual del efecto riqueza que tiene origen en intervenciones gubernamentales, numerosos países hayan instaurado herramientas de captación de plusvalías (Furtado, 1999; Smith y Gihring, 2006), apoyadas en “(...) la idea de que el gobierno local debe recuperar, en beneficio de la comunidad, al menos una parte de los beneficios que capturan los terrenos por efectos de obras públicas y normativa urbana" (Cuenya y Corral, 2011:4).

\section{Antecedentes de la literatura}

\section{Contexto internacional}

Dado que la red de transportes es un mecanismo clave para la definición del grado de conectividad entre las diversas zonas de una ciudad y sus áreas neurálgicas, los cambios en el sistema de transporte, así como la adición de nuevos medios o bien la extensión de redes ya existentes, introducen modificaciones al espacio urbano (Gatzlaff y Smith, 1993; Medda y Modelewska, 2011). Dichas alteraciones en el territorio, a su vez, generan modificaciones en términos de población circulante -e.g. congestión, ruidos-, pudiendo configurar modificaciones en los valores del suelo y de los inmuebles localizados en las proximidades de las estaciones. La cadena secuencial planteada refiere a la idea de que:
2. V.g. en las cercanías de lugares de trabajo, esparcimiento, aprovisionamiento, entre otros (Wagner, 2009:13). 
(...) la inversión pública beneficia en primer lugar a los hogares del barrio en el que se realiza la inversión, haciéndolo más atractivo. En consecuencia, más gente desea mudarse allí y la demanda de vivienda se incrementa. Esto eleva los precios y las rentas de las propiedades y, por consiguiente, los beneficios del proyecto generan hasta cierto grado un aumento del valor de las mismas (Laakso, 1992:219).

En la literatura es factible encontrar múltiples trabajos dedicados a la identificación y medición de la magnitud y dirección del impacto sobre precios de propiedades derivado de la cercanía a estaciones de redes de transporte alternativos. Su revisión permite establecer que no existe consenso definitivo en torno a la modalidad -y sentido- de incidencia de las inversiones en infraestructura urbana sobre el precio de las propiedades (Spengler, 1930; Gatzlaff y Smith, 1993). En efecto, los inmuebles próximos a estaciones de transporte público masivo se ven alcanzados por mecanismos diversos que operan sobre el espacio urbano, y suelen compensarse entre sí. Tal como señalara el Center for Urban Mobility Research:

el transporte público puede mejorar el valor de las propiedades cercanas, dotando de mejor accesibilidad y visibilidad, o puede deprimirlo a causa del ruido, la polución, los disturbios provocados por la construcción, aislamiento permanente de algunas viviendas, u otros efectos (Rice Center, 1987:1).

Un punto adicional a ser considerado en las investigaciones refiere a la multiplicidad de etapas que atraviesan al sistema de transporte, a saber: elección de la ruta, acondicionamiento del territorio, construcción, y funcionamiento. Por lo cual, entre las modalidades técnicas de evaluación de impacto más ampliamente difundidas destacan: a) la comparación entre valores de inmuebles previos y posteriores a la llegada del medio de transporte en cuestión; y b) el contraste entre valores de propiedades dentro y fuera de un radio de incidencia definido (Rice Center, 1987).

Damm et al. (1980) hacen uso de la metodología de precios hedónicos para evaluar los efectos del metro-rail en Washington, encontrando que los valores transaccionales de las propiedades cercanas a las estaciones analizadas son mayores, aunque el efecto distancia se agota rápidamente. Por su parte, Gatzlaff y Smith (1993) estudian el impacto del metro-rail en Miami a partir del análisis de índices de ventas recurrentes de inmuebles y la aplicación de regresiones hedónicas utilizando los últimos dos datos de precio de venta para una muestra de 912 inmuebles cercanos a 8 estaciones entre 1971 y 1990 e información de esas propiedades sobre superficie, fecha de construcción y dirección. Mediante la estimación hedónica, los autores encuentran evidencia de que las propiedades más cercanas tienen un precio relativo menor por la presencia de efectos de congestión, aquellas que se localicen a una distancia próxima aunque no inmediata también poseen un diferencial positivo. Los resultados dan cuenta de efectos sobre precios residenciales sensiblemente dependientes de la estación del metro-rail considerada, debido a lo cual los autores concluyen que: “(...) los barrios residenciales más caros han experimentado mayores incrementos en el valor de las propiedades cercanas a la estación de subterráneo mientras que esto no ha sucedido en los barrios menos atractivos" (Gatzlaff y Smith, 1993:64).

Asimismo, se destacan las investigaciones de Bajic (1983) sobre la evaluación de la influencia de una nueva línea de subterráneo en Toronto -con datos de 385 transacciones de inmuebles durante 1978-, de Downing (1973), sobre valores de las propiedades en Milwaukee-Wisconsin para el período 1958-1962, y en especial aquella de Cervero (1995), en la que se reseña el impacto sobre distintas metrópolis norteamericanas de diversos medios de transporte. Cervero encuentra un efecto positivo de hasta $4 \%$ sobre inmuebles a menos de 1000 pies de distancia del BART (Bay Area 
Rapid Transit) en San Francisco, lo cual contrasta con el hallazgo de una incidencia negativa de la cercanía al BART en los trabajos de Burkhardt (1976) y Dornbush (1975), reflejo de mayores niveles de ruido y tránsito.

Por su parte, en Laakso (1992) se examina el efecto de la construcción del metro en Helsinki sobre propiedades residenciales, mediante estimaciones de corte transversal en los años 1980, 1985 y 1989, siendo los primeros dos pre y el último post construcción del metro, encontrando que:
el precio de la propiedad ubicado a una distancia cercana a una estación de subte o tren es hasta un 7,5 por ciento mayor que en cualquier otro lado y advirtiendo que esos incrementos en los valores inmobiliarios resultan mayores para propiedades ubicadas a una distancia de entre 500 y 750 metros de la estación en el caso del subterráneo, y entre 250 y 500 metros en el caso del tren (Laakso, 1992:223).

Finalmente, Bowes e Ihlanfedlt (2001) encuentran para el caso de Atlanta la presencia de no-linealidades vinculadas al efecto proximidad, de manera que las propiedades ubicadas a menos de un cuarto de milla (equivalente a un radio de influencia de 400 metros) de la estación de tren presentan precios de oferta un 19\% inferiores a los correspondientes a inmuebles cuya distancia es más de 3 millas, al tiempo que las ubicadas entre una y tres millas tienen, en promedio, valores más elevados en relación a los inmuebles más alejados. Y Debrezion, Pels y Rietveld (2007) estiman el efecto distancia a partir de dos medidas: una, vinculada al impacto sobre propiedades en un rango de un cuarto de milla de la estación en cuestión, y la segunda, una medida de distancia en términos continuos; distinguiendo, a la vez, entre propiedades residenciales y comerciales. Los hallazgos implican que el efecto de proximidad es positivo: al utilizarse la primera medida de distancia, constatan precios $4,2 \%$ y $16,4 \%$ superiores para inmuebles residenciales y comerciales, respectivamente; en tanto que, para la medida de proximidad continua, encuentran que acercarse 250 metros a una estación eleva el valor de la propiedad en un $2,4 \%$ para residenciales, y sólo en $0,1 \%$ para comerciales.

A continuación, se presenta una revisión de los estudios dedicados a analizar la incidencia de la infraestructura urbana sobre el mercado inmobiliario en Argentina.

\section{Contexto local}

Por lo general, los estudios de caso aplicados en Argentina coinciden en el uso de la metodología de precios hedónicos a fin de cuantificar la valoración de ciertos atributos en el mercado inmobiliario. Dado que no existe disponibilidad de información respecto a la variable principal -el precio de venta- el criterio de los autores suele ser la consideración del precio de oferta de las propiedades como un buen proxy del valor final de venta.

De todas formas, como constituye una agenda de investigación no explorada en profundidad, se incluyen aquí estudios de precios hedónicos que examinan la contribución a los medios de transporte aún cuando éste no representara el objeto de análisis de la investigación. Tal es el caso de Conte Grand (2001), quien estudia los efectos de la contaminación en el valor de los inmuebles, y en su regresión hedónica propone como potencial regresora la distancia a las estaciones de subte, encontrando un efecto no significativo y directo (a mayor distancia, mayor precio). Este trabajo se efectúa únicamente para un barrio de la CABA, con lo cual hacia el final se plantea la necesidad de un estudio de mayor envergadura y detalle respecto a las posibles no linealidades que pudieran existir. 
Uno de los primeros abordajes no lineales relacionados con modelos de precios hedónicos se encuentra incluido en la contribución de Gómez Mera (1998), donde si bien se estudia el efecto de la cercanía a las plazas de la CABA, se ahonda en el concepto de área de influencia, identificando un coeficiente positivo y decreciente en un radio de 750 metros de distancia de los espacios verdes (Gómez Mera, 1998:16). Paralelamente, para el caso de la ciudad de San Miguel de Tucumán, Meloni y Ruiz Nuñez (1997) cuentan con los datos de una Encuesta de Valores Inmobiliarios sobre baldíos y terrenos, y analizan, entre otras, la relación entre el precio y la ubicación (cercanía a la zona comercial de la ciudad), encontrando un efecto positivo. Resulta interesante mencionar este trabajo, en primer lugar por constituir uno de los pocos estudios para Argentina que no se concentra en analizar la CABA, y en segundo lugar por mencionar un aspecto del análisis espacial no siempre incluido en esta línea de investigación: el testeo de dependencia espacial. ${ }^{3}$

En Castillo (2008), el análisis efectuado se asemeja al propuesto en este trabajo: el estudio de la influencia de la cercanía al subte por rangos. Allí se consideran inicialmente variables dicotómicas para las observaciones pertenecientes a la zona de un radio 0 a 400 metros de las líneas de subte, para, en una instancia posterior, verificar la robustez de los resultados a partir de la modificación de los radios. Los hallazgos dan cuenta de un efecto positivo de la cercanía al subte, aunque no lineal, observándose una disminución del efecto a medida que las propiedades se encuentran más lejos del mismo.

En consonancia con este trabajo, Fuks et al. (2012) construyen, asimismo, una estimación ubicando las propiedades a diferentes distancias de las bocas de subte. Los autores encuentran también la presencia de una no linealidad, si bien identifican un efecto negativo -en comparación con el resto de las propiedades cercanas al subte- en el hecho de tener una distancia menor a cien metros de la boca de subte.

Una de las contribuciones más recientes Orduna et al. (2016) se propone estudiar el cambio en los precios de las propiedades producto de la puesta en funcionamiento de la primera línea de Metrobús, en la CABA. Aquí también se incluye la noción de área de influencia, considerando un efecto no lineal hasta un radio de 1000 metros desde la traza del metrobús, así como la contextualización del análisis en el marco de la identificación de las plusvalías urbanas. Los autores encuentran que el efecto es negativo en el precio, lo cual es opuesto a su expectativa, y por tanto proponen continuar con tal agenda de trabajo (Orduna et al., 2016:31).

En este contexto, nuestra contribución apunta a un doble objetivo. A saber, a) profundizar la agenda de investigación relacionada con los efectos de las políticas urbanas sobre localización de las propiedades, el efecto en sus precios y la potencial captación de plusvalías urbanas, y b) implementar modelos de regresión que den cuenta del carácter espacial y georreferenciado del objeto de estudio mediante un enfoque relativamente reciente -modelos con dependencia espacial- que ha tenido pocas aplicaciones en Argentina.

\section{Red de subterraneos de la Ciudad Autonoma de Buenos Aires}

Buenos Aires fue la primera metrópoli de Sudamérica en contar con red de transporte subterráneo. Cerca del centenario argentino, en el año 1909, la Compañía de Tranvías Anglo Argentina (CTAA), de capitales mixtos locales e ingleses, inicia la construcción de la primera de las 6 líneas que conforman la actual red de transporte metropolitano de la Ciudad Autónoma de Buenos Aires. 
La Línea A se inauguró luego de cuatro años de comenzadas las obras, en 1913, con un recorrido inicial entre Plaza de Mayo y Plaza Miserere, siendo extendida primero hasta la estación Río de Janeiro y luego hasta Caballito, en 1914. En tanto, la segunda línea, Línea B, cuyo recorrido iba entre las estaciones Chacarita y Callao, se inauguraría 16 años después, en 1930. Las obras correspondientes a las líneas subsiguientes, Líneas C y D, fueron realizadas por la Compañía Hispano Argentina de Obras Públicas y Finanzas (CHADOPyF), de capitales españoles y argentinos.

En el año 1944 se inauguraba la Línea E para el trayecto Constitución-General Urquiza. En el año 1991, y hasta la actualidad, la empresa Metrovías se hizo cargo de la concesión de la totalidad de las líneas. ${ }^{4}$ Asimismo, en 2012 se traspasa el control de dicha concesión desde el ámbito del Gobierno Nacional al Gobierno de la Ciudad Autónoma de Buenos Aires, que asumió formalmente la responsabilidad del servicio público de transporte mediante la promulgación de la Ley $4472,{ }^{5}$ norma que definió a la empresa Subterráneos de Buenos Aires S.E. (S.B.A.S.E) como autoridad de aplicación.

La última línea enteramente construida -e inaugurada en 2007-, Línea H, une Las Heras y Hospitales. Finalmente, en el año en 2008 se inauguraron las estaciones Puan y Carabobo de la Línea A; en 2013, dos estaciones que prolongaron la Línea B hasta el barrio de Villa Urquiza, y las últimas dos estaciones de la Línea A, que incorporaron cerca de 3 kilómetros al recorrido previo, San José de Flores y San Pedrito (Figura 1).

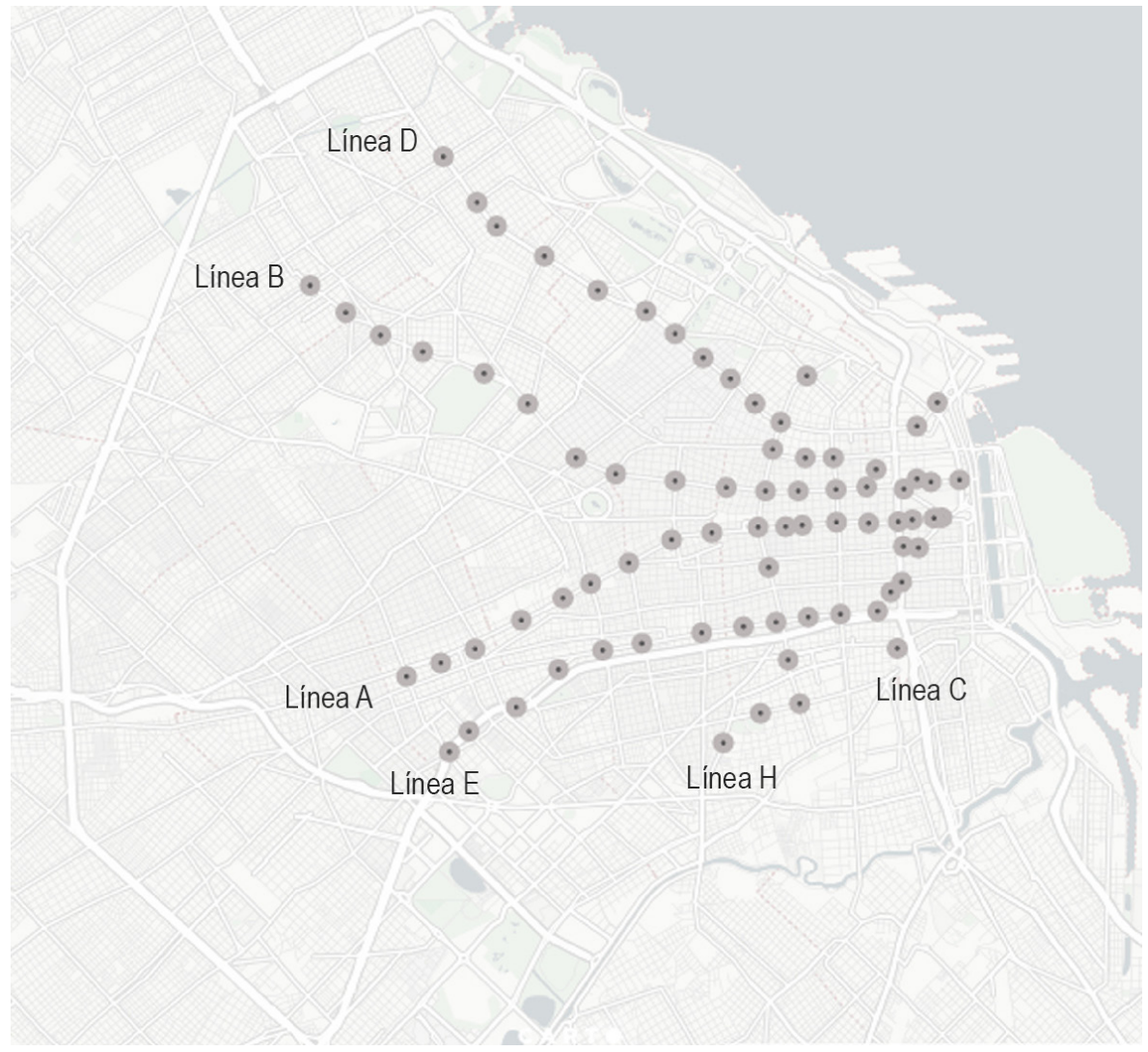

Figura 1. Cobertura de la Red de Subterráneos de la Ciudad de Buenos Aires. Fuente: Elaboración propia en base a Buenos Aires Data. Gobierno de la Ciudad de Buenos Aires.

En suma, la red de transporte subterráneo metropolitano en funcionamiento cuenta con 6 líneas, 86 estaciones, y una extensión agregada de 53,7 km. En el Cuadro 1 se presenta la información por línea, mientras que de la Figura 1 se deprende la existencia de zonas con grado de conectividad diverso.
4. Inicialmente, la concesión vencía el 31 de diciembre del 2017. Sin embargo, la legislatura porteña aprobó la extensión hasta diciembre del 2018, momento a partir del cual se deberá convocar a una nueva licitación para la renovación de la concesión del servicio por un nuevo lapso de 12 años -con potencial extensión de 3 años más-. Véase: http://www.ambito. com/902331-subte-extienden-porun-ano-la-concesion-a-metrovias.

Consultado el 26/02/2018.

5. Refiérase a http://www2. cedom.gov.ar/es/legislacion/ normas/leyes/ley4472.html. Consultado el 26/02/2018. 
Cuadro 1. Datos de la Red de Subterráneos de la Ciudad de Buenos Aires. Fuente: Elaboración propia en base a Metrovías.

\begin{tabular}{|l|c|c|c|c|c|c|}
\hline Línea & Año & Recorrido inicial & Recorrido actual & Duración (min) & Longitud (km) & Estaciones \\
\hline A & 1913 & Plaza de Mayo - Plaza Once & Plaza de Mayo - San Pedrito & 26 & 9,8 & 18 \\
\hline B & 1930 & Callao - F. Lacroze & Leandro N. Alem - J. M de Rosas & 27 & 11,9 & 17 \\
\hline C & 1934 & C. Pellegrini - Constitución & Retiro - Constitución & 13 & 4,5 & 9 \\
\hline D & 1937 & Florida - Tribunales & Catedral - C. de Tucumán & 26 & 10,4 & 16 \\
\hline E & 1944 & Constitución - Gral. Urquiza & Bolívar - Plaza de los Virreyes & 24 & 9,6 & 15 \\
\hline H & 2007 & Once - Caseros & Las Heras - Hospitales & 18 & 7,5 & 11 \\
\hline
\end{tabular}

\section{Heterogeneidades en el mercado de suelo urbano}

Durante las últimas décadas la dinámica inmobiliaria de la Ciudad Autónoma de Buenos Aires no resultó ajena a los procesos de volatilidad económica y política que atravesó Argentina, fundamentalmente a partir del período de Convertibilidad.

En la década de 1990, la idea de convertir a Buenos Aires en una ciudad global (Sassen, 1991) estuvo signada por el surgimiento de formas de urbanización privadas (i.e. countries, barrios privados). Las nuevas formas de generación de espacios de urbanos se dio en forma sincrónica con un proceso de retracción del Estado, marcado por la dinámica de privatización de servicios urbanos -e.g. servicios públicos de agua, luz, y de transporte, como trenes y subterráneo- (Prevot Schapira, 2002), que contribuyó a acentuar las diferencias territoriales heredadas.

Como corolario Szajnberg (2006) señala la emergencia de nuevos actores y objetos urbanos -e.g. shoppings, hoteles cinco estrellas-, que incidieron sobre una redefinición de pautas de producción y consumo del espacio residencial. Además, es durante este período que "las tendencias de localización de los emprendimientos residenciales habría continuado y profundizado la lógica preexistente de densificación de los corredores norte y oeste" (Szajnberg, 2006:76).

Múltiples factores explican la actual distribución espacial de precios del suelo. La preponderancia de superficie construida sobre el corredor norte en la década de 1970 y la intensificación de las preferencias de demanda por la calidad de infraestructura urbana en el norte de la Ciudad durante la década de 1990 ponen de relieve la distinción tradicionalmente efectuada entre Zona Norte y Zona Sur, en términos de desempeño de indicadores: habitacionales, demográficos, de salud, educativos, laborales e ingresos y de condiciones de vida (Gobierno de la Ciudad de Buenos Aires, 2006).

Tras el abandono de la paridad peso-dólar se producen nuevas dinámicas inmobiliarias, entre las que destaca un notable proceso de valorización del suelo, cuyo precio creció por encima del nivel general de precios de la economía (Baer y Kauw, 2016). Dicho proceso de valorización no resultó homogéneo al interior de la ciudad, como se evidencia en la Figura 2, sino que contribuyó a profundizar las disparidades de valores inmobiliarios entre Zona Norte, Zona Centro y Zona Sur. Por su parte, la relación de valores entre los distintos corredores se expone territorialmente, para el año 2018, en la Figura 3. 


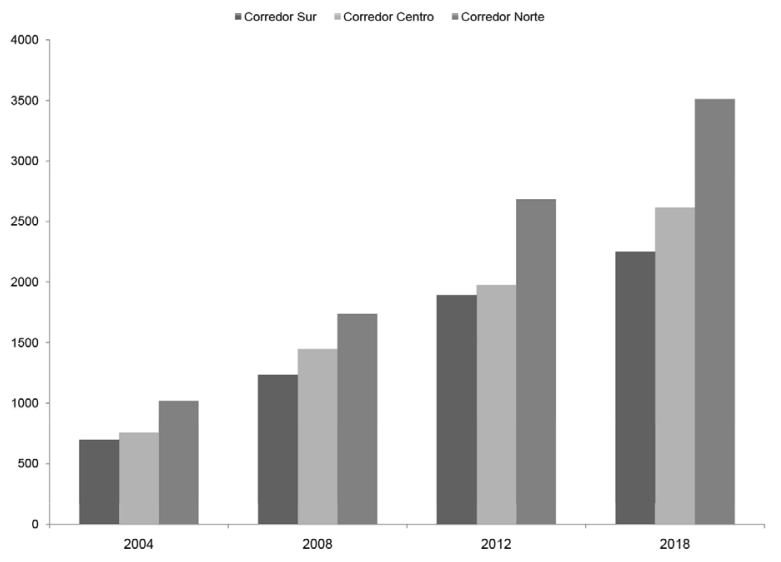

Figura 2. Evolución Precio por metro cuadrado de departamentos de o a 20 años en venta por Corredor Dólares corrientes. Fuente: Elaboración propia. Nota: Para todos los años se toman los corredores conformados para 2018. Los barrios que conforman cada corredor son los siguientes. Corredor Norte: Nuñez, Belgrano, Coghlan, Villa Urquiza, Parque Chas, Villa Ortúzar, Belgrano, Colegiales, Palermo, Villa Crespo, Caballito, Almagro, Recoleta, Retiro, San Nicolás, Puerto Madero y San Telmo ; Corredor Centro: Saavedra, Villa Pueyrredón, Villa Devoto, Monte Castro, Agronomía, Villa del Parque, Paternal, Chacarita, Villa Santa Rita, Villa Gral. Mitre, Flores, Parque Chacabuco, Boedo (desde Av. San Juan hacia el Norte), San Cristóbal, Balvanera, Monserrat, Barracas, y Corredor Sur: Villa Real, Versalles, Liniers, Villa Luro, Velez Sarsfield, Floresta, Mataderos, Parque Avellaneda, Villa Lugano, Villa Riachuelo, Villa Soldati, Pompeya, Boedo (desde Av. San Juan hacia el Sur), Parque Patricios, Constitución, La Boca.

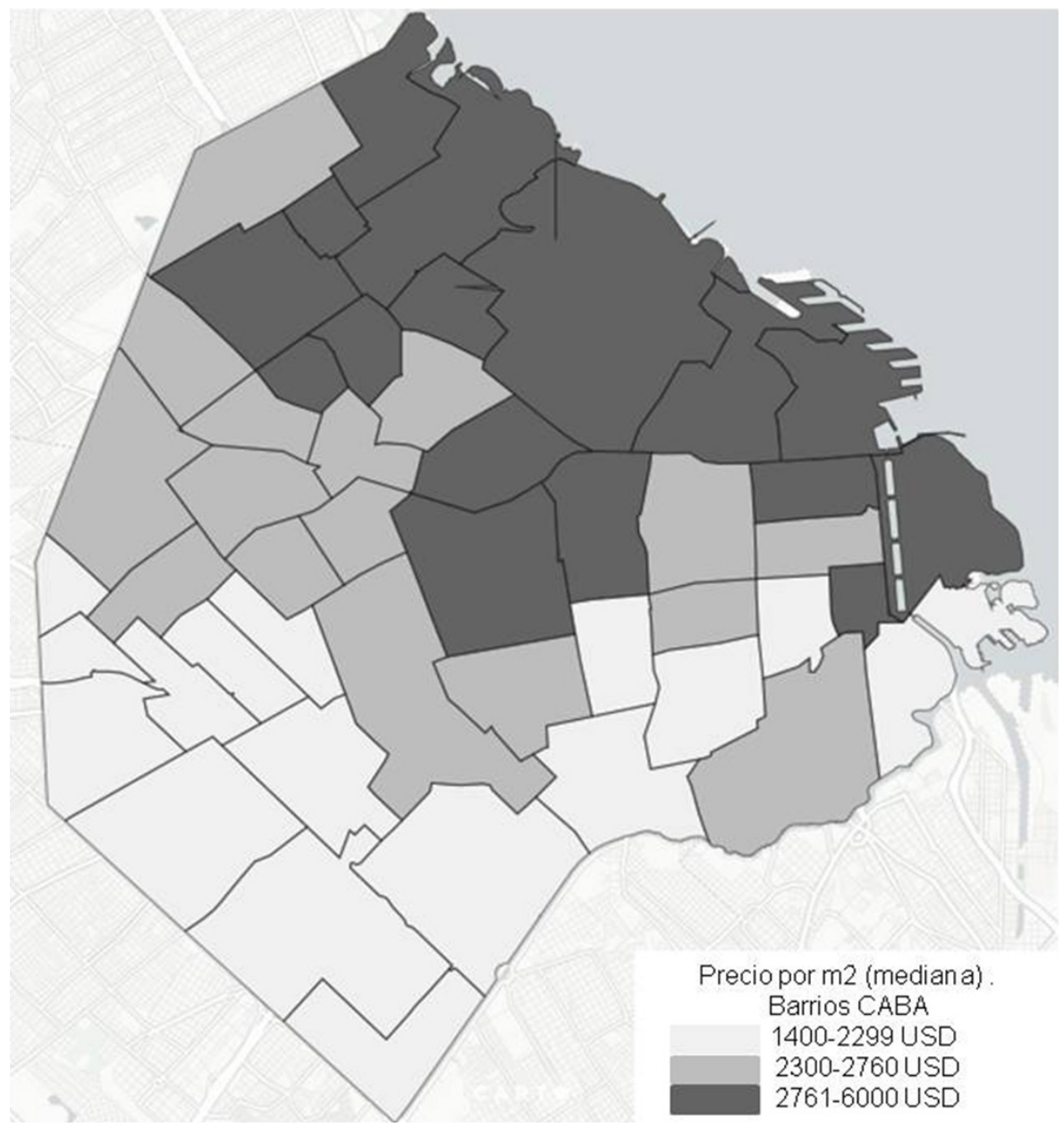

Figura 3. Mediana del precio por metro cuadrado de departamentos de o a 20 años en venta por barrio - Ciudad Autónoma de Buenos Aires. Dólares corrientes. Año 2018. Fuente: Elaboración propia. 
A tal efecto, se excluyeron del análisis las estaciones de cada una de las líneas correspondientes a zonas de uso no estrictamente residencial, tomando como referencia las especificaciones contenidas en el Código de Planeamiento Urbano 2017, ${ }^{6}$ ubicadas en áreas oficinales. De modo que fueron consideradas:

» Línea A: Loria, Castro Barros, Rio de Janeiro, Acoyte, Primera Junta, Puan, Carabobo, Flores, San Pedrito;

» Línea B: Carlos Gardel, Medrano, A. Gallardo, Malabia, Dorrego, F. Lacroze, Tronador, Incas, E. Echeverría, J. M. de Rosas;

» Línea C: Retiro, San Martin, San Juan, Constitución;

» Línea D: Pueyrredón, Agüero, Bulnes, Scalabrini Ortiz, Plaza Italia, Palermo, Ministro Carranza, Olleros, J. Hernández, Juramento, C. de Tucumán.

» Línea E: Entre Ríos, Pichincha, Jujuy, Gral. Urquiza, Boedo, Av. La Plata, J. M Moreno, E. Mitre, M. Milagrosa, Varela, Plaza de los Virreyes.

» Línea H: Las Heras, Santa Fe, Córdoba, Venezuela, Humberto I, Inclán, Caseros, Parque Patricios, Hospitales.

En base a tal especificación, se calcularon los precios promedio por metro cuadrado para un radio de 600 metros de proximidad a cada una de las estaciones de la red de subterráneos (Cuadro 2). Se evidencia una significativa dispersión de guarismos entre líneas, pero también entre las diversas bocas que conforman cada ramificación de la red de subterráneo. Dichas relaciones entre líneas de subte pueden observarse en la Figura 4.

Cuadro 2. Precio por metro cuadrado de departamentos de o a 20 años en las cercanías de las Líneas de Subterráneo de la Ciudad Autónoma de Buenos Aires. Promedio Simple y Desvío Estándar. Estaciones Residenciales. Dólares corrientes. Inmuebles de o a 20 años en venta entre Septiembre y Diciembre de 2017, localizados a menos de 600 metros de distancia de las bocas de subte. Fuente: Elaboración propia.

\begin{tabular}{|l|c|c|}
\hline Línea & Promedio & Desvío \\
\hline $\mathrm{A}$ & $2.582,85$ & 302,43 \\
\hline $\mathrm{B}$ & $2.811,92$ & 217,51 \\
\hline C & $3.205,75$ & $1.035,86$ \\
\hline $\mathrm{D}$ & $3.774,06$ & 367,42 \\
\hline E & $2.349,69$ & 447,02 \\
\hline H & $2.586,06$ & 721,09 \\
\hline
\end{tabular}

Así, el menor valor medio del $\mathrm{m}^{2}$ (USD 2349,69) se corresponde con los inmuebles ubicados en las proximidades de la línea E, que recorre la zona sur de la ciudad, de este a oeste; al tiempo que el máximo se encuentra para los alrededores de la Línea D (USD 3774,06), cuyo trayecto también va de este a oeste, pero atravesando la zona norte de CABA.
6. Véase http://www.ssplan.buenosaires.gov.ar/webfiles/mapa_cpu. php. Consultado el 26/02/2018. 


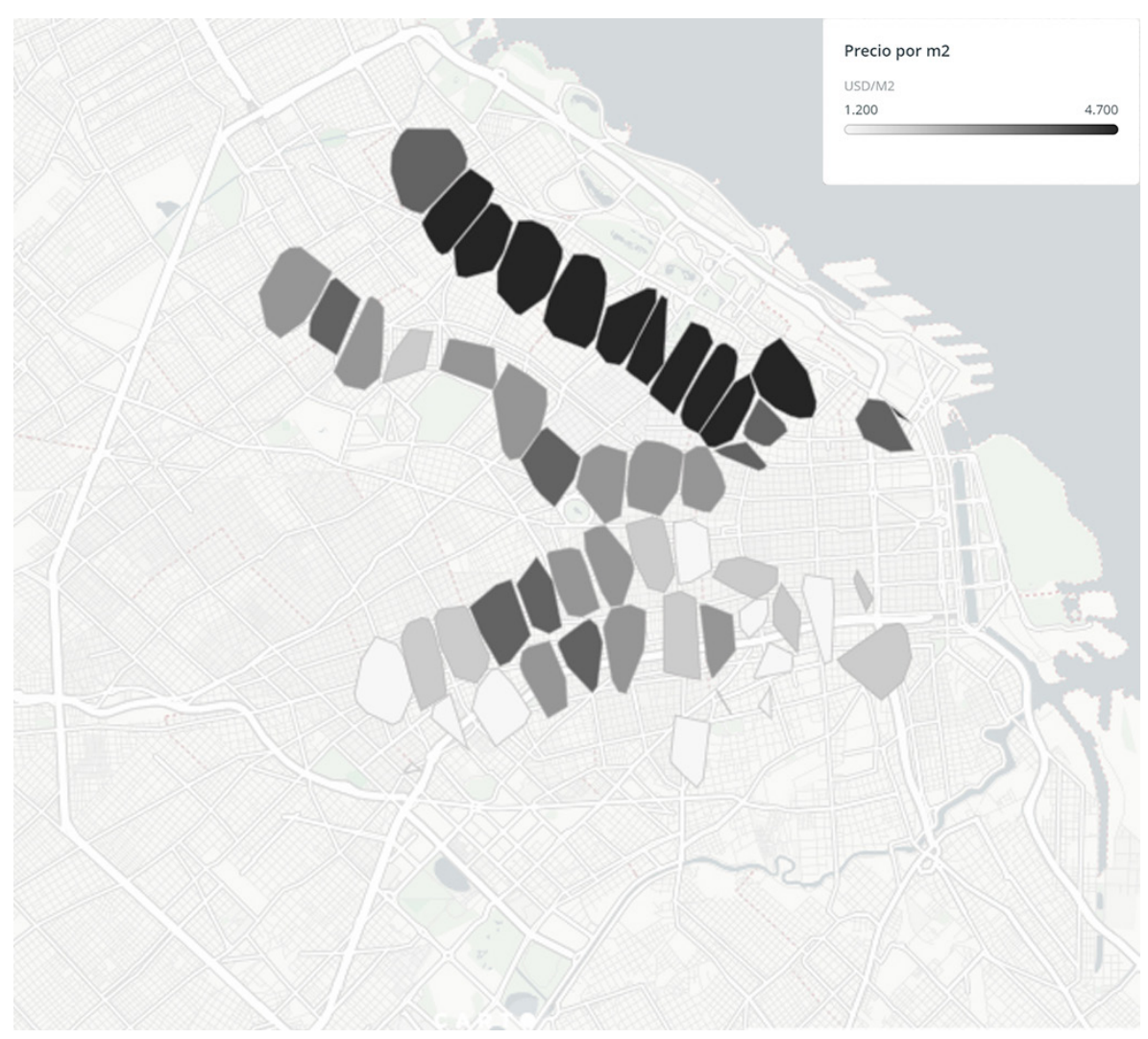

Figura 4. Precio por metro cuadrado de departamentos de o a 20 años en venta en un radio de 600 metros de las Líneas de Subterráneo de la Ciudad Autónoma de Buenos Aires. Promedio Simple.

Estaciones Residenciales. Dólares corrientes. Fuente: Elaboración propia.

En términos promedio, el valor del metro cuadrado para inmuebles en venta cercanos a la Línea $\mathrm{D}$ resulta un $60,1 \%$ superior al de inmuebles contiguos a la Línea $\mathrm{E}$, un $46,1 \%$ por encima de los de la línea $\mathrm{A}$, un $45,9 \%$ respecto a los de la línea $\mathrm{H}$, un $34,2 \%$ en relación a los de la línea B, y un $17,7 \%$ por encima de los de la línea C. Asimismo, se verifica que en el rango entre $1200 \mathrm{USD} / \mathrm{m}^{2}$ y $4700 \mathrm{USD} / \mathrm{m}^{2}$, las estaciones que reportan valores más elevados se corresponden con la Línea $\mathrm{D}$, a la vez que se pone de manifiesto la presencia de heterogeneidades entre las distintas bocas que conforman cada una de las 6 líneas (Figura 5). 

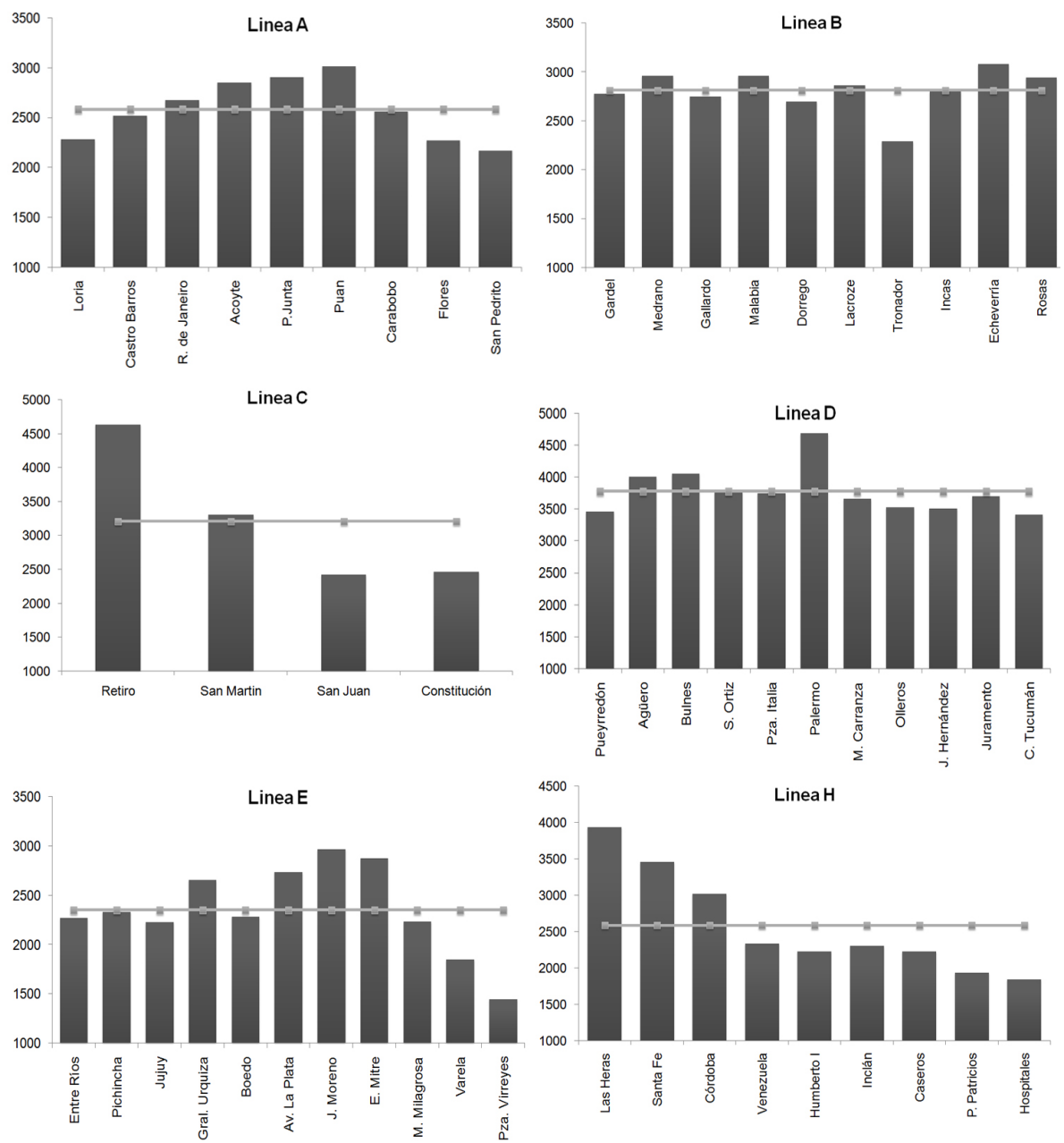

Figura 5. Precio del metro cuadrado de la Red de Subterráneos de la Ciudad Autónoma de Buenos Aires, por estación (barras) y Línea (línea). Promedio simple. Estaciones Residenciales. Inmuebles de o a 20 años en venta entre Septiembre y Diciembre de 2017, localizados a menos de 600 metros de distancia de las bocas de subte. Fuente: Elaboración propia.

Del análisis más detallado al interior de las distintas líneas, se destaca que: a) para el caso de aquellas que atraviesan verticalmente la ciudad, Líneas $\mathrm{H}$ y $\mathrm{C}$, el valor del $\mathrm{m}^{2}$ promedio de las propiedades contiguas a las bocas del subte desciende al avanzar hacia la zona sur, reforzando la idea previamente señalada; b) la línea B presenta la menor dispersión de valores entre las propias estaciones constitutivas; c) para las líneas A y $\mathrm{E}$, los valores más altos se hallan en torno a estaciones ubicadas en el extremo final de la línea, evidenciándose una baja en el precio para las 3 estaciones más alejadas del centro de referencia de la CABA (marcado verticalmente por la Avenida 9 de Julio); d) en relación a la línea $\mathrm{D}$, destaca la superioridad de los valores ligados a todas las estaciones consideradas, y, entre la propia línea, el correspondiente a la estación Palermo.

A partir de lo anterior cabría pensar que las zonas más caras conjugan un relativo alejamiento, pero a la vez buena conectividad en términos de distancia medida en estaciones de subte que implica el desplazamiento hacia la zona céntrica y de oficinas: para Línea A, estaciones P. Junta y Puan; para la E, Moreno y Mitre.

Ahora bien, con miras a profundizar el examen en lo que refiere al impacto de la infraestructura urbanística de transporte sobre los valores transaccionales de inmuebles, $\mathrm{y}$, 
habiéndose descripto las diferencias entre líneas y entre estaciones al interior de una misma línea, el siguiente paso es analizar la incidencia de la proximidad a una boca de subte sobre el precio. Y de manera concomitante, el examen referido a la existencia y magnitud de efectos no lineales entre distancia al subte y valor de mercado del inmueble. Se pretende echar luz sobre cierta noción presente en el sentido común de la sociedad referida a la naturaleza de un efecto riqueza sobre las propiedades que se desprende de la decisión y acción gubernamental de emplazamiento de infraestructura urbana en el territorio. Se describe a continuación el proceso metodológico, y, luego, los resultados obtenidos al respecto.

\section{Metodologia y fuentes de informacion}

En el marco del análisis del mercado inmobiliario, un hecho común a otras investigaciones es la necesidad de utilizar como proxy del precio de venta al precio de oferta. Nuestra base de datos comprende 8098 observaciones georreferenciadas de avisos de venta de departamentos en la Ciudad de Buenos Aires, recolectadas de sitios de internet para el período comprendido entre septiembre y diciembre del 2017. Junto con el precio de venta, se incorporan variables que caracterizan a la propiedad (superficie, barrio, antigüedad, ubicación respecto a las bocas de subte más cercanas, presencia de amenities en el edificio, entre otras). Cabe señalar que las observaciones se encuentran distribuidas en todo el territorio de la ciudad, y no únicamente en las cercanías a la red de subterráneos.

El modelo de regresión a implementar busca cuantificar la relevancia que detentan diversas clases de atributos respecto al precio de venta de la vivienda, siguiendo la metodología de precios hedónicos (Rosen, 1974; Sheppard, 1999) e incluyendo en el análisis la potencial presencia de efectos espaciales (Anselin, 2013).

La técnica de precios hedónicos consiste en mensurar el efecto sobre el precio de un bien transado en el mercado atribuible a las características del mismo. El mercado inmobiliario presenta la particularidad de enmarcar transacciones que comprenden al bien final, aunque es factible identificar la valoración implícita por sus atributos específicos. El precio de una vivienda puede ser explicado a partir de los atributos de esa unidad, distinguiendo entre atributos del inmueble per se, y atributos de localización (Goodman, 1978:472). En nuestro caso, dados los atributos considerados, el precio de la vivienda $i, P_{v i}$ puede expresarse en función del set de atributos del inmueble $A_{l j}$, con $j=1, \ldots, n$; de los atributos del edificio $A E j$, con $j=1, \ldots, n$; y del set de atributos de localización $A L j$, con $j=1, \ldots, n$.

$$
P_{v i}=P_{v}\left(A I_{1}, \ldots, A I_{n} ; A E_{1}, \ldots, A E_{n} ; A L_{1}, \ldots, A L_{n}\right),
$$

El precio marginal de cada uno de los atributos se obtendrá a partir de la diferenciación de la función de precios implícitos respecto a esas características. Formalmente, para los atributos de localización, los coeficientes obtenidos responderán a:

$$
\frac{\delta P_{v i}}{\delta A L_{j}}=P_{A L_{j}}\left(A L_{j}\right)
$$


y su interpretación debe entenderse como el aumento/descenso en el precio de la vivienda que se deriva del cambio en una unidad del atributo considerado, ceteris paribus (Freeman, 1979).

En particular, para el análisis efectuado se consideraron:

" Atributos del inmueble: superficie total, antigüedad (distinguiendo entre usados, por rangos de antigüedad, y a estrenar), cochera, orientación al frente, lavadero, amoblado.

»Atributos del edificio: pisos del edificio, pileta, gimnasio, vigilancia.

»Atributos de localización/conectividad: se calculó la distancia euclídea -en línea recta- entre el par de coordenadas asociado a cada uno de los inmuebles que conforman la base de datos y los correspondientes a la totalidad de las bocas de la red subterránea de la Ciudad Autónoma de Buenos Aires. A partir de tal ejercicio, se construyeron variables dummies de proximidad a este medio de transporte, a efectos de evaluar no-linealidades. A saber: a) muy próximo al subte, inmuebles en el rango 0-200 metros del subte; b) próximo al subte, inmuebles en el rango 200-300 metros del subte; c) cercano al subte, inmuebles en el rango 300-600 metros del subte; d) en el potencial radio de influencia del subte, inmuebles en el rango 0-600 metros del subte.

En relación a la determinación del área de influencia -buffer- del medio considerado sobre los valores de los inmuebles, se adoptó la categorización aludida precedentemente, tomando como referencia: a) el criterio utilizado por Castillo (2008) y retomado en Orduna et al. (2016), que establece una incidencia del efecto proximidad significativa para propiedades ubicadas en un rango de 0 a 800 metros en línea recta; b) el radio previsto en la Ley de Subterráneos - Fondo Permanente para la ampliación de la red (Ley 23.514/1987), de 400 metros de tránsito directo a la boca de acceso a cada línea; y c) Indicador de proximidad de paradas a transporte público de CAT-MED, Platform for Sustainable Urban Models, ${ }^{7}$ que define el ámbito de proximidad deseable para paradas de metro en 500 metros.

A los efectos de analizar la influencia de la antigüedad del inmueble sobre el precio del metro cuadrado, se construyeron variables dummy a partir de la variable antigüedad medida en años. Así, por un lado se distingue entre inmuebles usados y a estrenar; y por otro, entre las propiedades usadas, se crean variables correspondientes a distintos rangos de antigüedad (en años): de 1 a 10; de 11 a 20, de 21 a 30, y superior a 41; tomando como base de comparación al grupo de propiedades entre 31 y 40 años de antigüedad.

Finalmente, se construyeron dos variables dicotómicas para identificar a los barrios en los cuales se concentran los valores extremos de la variable dependiente. En particular, se identificaron los barrios cuyo promedio de precio/ $\mathrm{m}^{2}$ se ubicara en el $10 \%$ superior (v.g. Puerto Madero) e inferior (v.g. Villa Lugano-Villa Soldati; Parque Patricios) de la distribución. En el Cuadro 3 se presenta un resumen estadístico de las variables que caracterizan a las propiedades.
7. http://www.catmed.eu/dic/ es/52/proximidad-a-paradasde-transporte-publico. Consultado el 26/02/2018. 
Cuadro 3. Estadísticas descriptivas sobre variables de caracterización de las observaciones." Para el caso de los valores expresados originalmente en pesos, la conversión a dólares se realizó tomando el valor de referencia informado por el Banco Central de la República Argentina de la semana de publicación del aviso en cuestión. Fuente: Elaboración propia.

\begin{tabular}{|l|c|c|c|c|c|c|c|}
\hline Variable & Media & Desvío & Min & Max & Mediana & $\begin{array}{c}\text { Clase de } \\
\text { Atributo }\end{array}$ & Especificación \\
\hline Precio del $\mathrm{m}^{2}$ & 2876,11 & 1044,38 & 589,04 & 9932,99 & 2683,129 & inmueble & en USD \\
\hline Superficie & 92,789 & 74,636 & 21 & 800 & 68 & inmueble & $\mathrm{m}^{2}$ \\
\hline A estrenar & 0,1365 & 0,3433 & 0 & 1 & 0 & inmueble & dummy \\
\hline Antigüedad 1-10 años & 0,1851 & 0,3884 & 0 & 1 & 0 & inmueble & dummy \\
\hline Antigüedad 11-20 años & 0,0727 & 0,2597 & 0 & 1 & 0 & inmueble & dummy \\
\hline Antigüedad 21-30 años & 0,0675 & 0,251 & 0 & 1 & 0 & inmueble & dummy \\
\hline Antigüedad +41 años & 0,273 & 0,4455 & 0 & 1 & 0 & inmueble & dummy \\
\hline Pisos & 10,026 & 6,561 & 2 & 52 & 9 & edificio & $\mathrm{n}^{\circ}$ de pisos del edificio \\
\hline Pileta & 0,227 & 0,419 & 0 & 1 & 0 & edificio & dummy \\
\hline Cochera & 0,418 & 0,493 & 0 & 1 & 0 & inmueble & dummy \\
\hline Gimnasio & 0,126 & 0,332 & 0 & 1 & 0 & edificio & dummy \\
\hline Frente & 0,699 & 0,459 & 0 & 1 & 1 & inmueble & dummy \\
\hline Vigilancia & 0,195 & 0,396 & 0 & 1 & 0 & edificio & dummy \\
\hline Lavadero & 0,67 & 0,47 & 0 & 1 & 1 & inmueble & dummy \\
\hline Amoblado & 0,03 & 0,17 & 0 & 1 & 0 & inmueble & dummy \\
\hline
\end{tabular}

En base a la secuencia metodológica descripta, se estimaron modelos de regresión hedónica semi-logarítmica, en los que la variable dependiente se encuentra expresada en términos logarítmicos. Por consiguiente, los coeficientes, en todos los casos, deben interpretarse como semi-elasticidades. En particular, se tomaron en cuenta dos especificaciones alternativas:

(A) Especificación con rangos de proximidad:

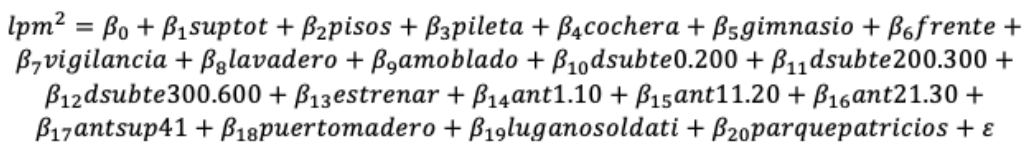

(B) Especificación sin rangos de proximidad:

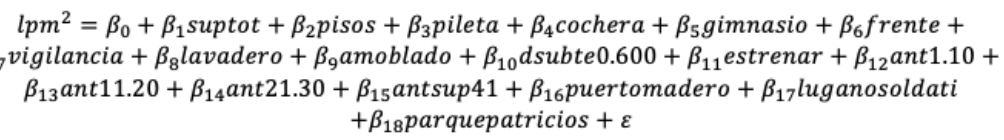

Una particularidad de este tipo de información es que se encuentra distribuida espacialmente en una misma ciudad. Por este motivo, en la literatura de modelos hedónicos para datos georreferenciados se hace especial hincapié en la posibilidad de existencia de dependencia espacial, cuya consecuencia más estudiada es la autocorrelación espacial. ${ }^{8}$ Anselin y Bera (1998) definen a la autocorrelación espacial como un caso especial de dependencia cross-section, vinculada al patrón que toma una variable en relación su localización.

Si no se puede asegurar que las observaciones sean independientes, resulta necesario evaluar si se encuentran espacialmente correlacionadas. De lo contrario, los parámetros estimados serán ineficientes y podrían parecer estadísticamente no significativos. ${ }^{9}$ En el caso del mercado inmobiliario, nuestra presunción es que existe dependencia espacial, dado que si bien pueden existir heterogeneidades al interior de los barrios, éstas

8. A diferencia de las estructuras de datos de series de tiempo o de panel, en las cuales la autocorrelación temporal tiene una única dirección, en este caso debemos contemplar todas las direcciones posibles.

9. En particular, los estimadores obtenidos por MCO en presencia de un rezago espacial de la variable dependiente serán sesgados, en tanto que en el caso de autocorrelación espacial en los residuos de la regresión, los estimadores serán ineficientes (Anselin, 1988, p. 57 y ss.). 
serán menores que en una comparación inter-barrial. De modo que es factible que los precios de la vivienda se encuentren agrupados en un proceso no aleatorio. Esta presunción será uno de los primeros elementos a contrastar.

Todas las pruebas de dependencia espacial requieren la construcción de una matriz de ponderadores espaciales (W), en la cual se resuma la información de la distancia o contigüidad entre las observaciones (Fischer y Nijkamp, 2014). Esta matriz es cuadrada y simétrica, de dimensión $n x n$, y en cada elemento $a_{i j}$ puede encontrarse, o bien la distancia entre la unidad $i$ y la unidad $j$, o bien un valor 1 para todas las unidades que sean vecinas (Herrera, 2015). Formalmente,

$$
\boldsymbol{W}=\left[\begin{array}{ccc}
a_{i j} & \cdots & a_{i n} \\
\vdots & \ddots & \vdots \\
a_{n j} & \cdots & a_{n n}
\end{array}\right] \text { con } \begin{aligned}
& a_{i j}=0 \text { si } i=j \\
& a_{i j} \neq 0 \text { si } i \neq j
\end{aligned}
$$

En nuestro caso, los elementos de la matriz miden la distancia inversa entre observaciones, lo cual permite capturar de manera más precisa los distintos grados de cercanía entre observaciones (Getis, 2009). Formalmente,

$$
a_{i j}=\frac{1}{\left(\left|l a t_{i}-l a t_{j}\right|^{2}+\left.\left|l n g_{i}-\operatorname{lng}\right|_{j}\right|^{2}\right)^{1 / 2}},
$$

Adicionalmente, se pueden truncar los valores para asignar un ponderador cero a las unidades que se encuentren a una distancia mayor a cierto valor. Para este trabajo se tomó una cota de $2 \mathrm{~km}$ (en términos de distancia euclídea inversa, 48,26). Finalmente, se utilizó la normalización espectral (i.e. cada elemento se divide por el mayor autovalor de la matriz) para estandarizar la matriz (Drukker et al., 2013). De esta forma se mantiene el carácter simétrico de la misma. Una vez construida la matriz de 8098 x 8098, pueden implementarse medidas de contrastación de dependencia espacial. Entre las más utilizadas en este tipo de investigaciones se encuentra el test I de Moran (1950). El método de evaluación consiste en correr la regresión inicial por Mínimos Cuadrados Ordinarios (OLS) y luego realizar un test de tipo Multiplicadores de Lagrange Robustos (Test LM) sobre los residuos a fin de determinar qué modelo de regresión espacial se debe implementar: Modelo de error espacial (SEM), Modelo de rezago espacial (SLM) o Modelo autorregresivo con residuos autorregresivos (SARAR). Los primeros dos pueden pensarse como un caso particular del siguiente modelo reducido (Anselin et al., 1996).

$$
\begin{aligned}
& \boldsymbol{y}=\lambda \boldsymbol{W} \boldsymbol{y}+\beta \boldsymbol{X}+\boldsymbol{u}, \\
& \boldsymbol{u}=\boldsymbol{\rho} \boldsymbol{W u}+\boldsymbol{\varepsilon}, \quad \text { siendo } \quad \boldsymbol{\varepsilon} \sim N\left(0, \sigma^{2}\right),
\end{aligned}
$$

A partir del cual se puede pensar que,

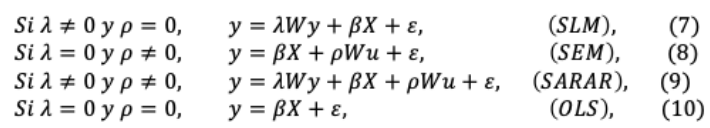


En estos tipos de modelos - en particular, SLM y SARAR -, una diferencia fundamental respecto a la regresión por OLS es que el efecto parcial de un incremento unitario de una variable independiente no será igual para todas las observaciones de la variable dependiente (Elhorst, 2014). Por el contrario, ante la presencia de dependencia espacial, su efecto total será el resultado de sumar el efecto directo sobre esa observación particular más el efecto indirecto a raíz de las unidades vecinas. Esta característica ha sido denominada efecto multiplicador, y su efecto decrece con la distancia.

En contraste, para el modelo SEM, si bien no se da tal rasgo, existe lo que se llama efecto difusión, asociado al término de error. Este implica que todo shock aleatorio sobre una unidad $i$ no solo afecta a la variable explicada en esa ubicación, sino también en el resto de las unidades, con un impacto decreciente con la distancia (De Gallo, 2014).

Cuando se rechazan las hipótesis nulas resultantes del Test Multiplicador de Lagrange Robusto para SLM y SEM, como en nuestro caso, existen dos alternativas: a) realizar una estimación del modelo SARAR; y b) comparar los resultados de los dos tests LM robustos a efectos de seleccionar el modelo (SLM o SEM) que presente un mayor nivel de significatividad (Anselin y Florax, 1995). La evidencia para esta muestra - tanto para la regresión con rangos de no linealidad como para la que no tiene diferenciación por distancia - parece indicar que el modelo de regresión espacial que mejor captura la autocorrelación espacial es el Modelo autorregresivo con residuos autorregresivos (SARAR), aunque también se estimará el Modelo de error espacial.

No obstante, tales modelos no pueden estimarse mediante MCO, dado que los coeficientes estimados resultarán inconsistentes y/o ineficientes. ${ }^{10}$ Uno de los métodos de estimación para este caso es el Método de Máxima Verosimilitud, cuyos estimadores son consistentes, eficientes y asintóticamente normales incluso en presencia de dependencia espacial en el error o en la variable dependiente. ${ }^{11}$

\section{Resultados}

De acuerdo a lo expuesto en la sección precedente, se consideran las especificaciones: (A) con rangos de proximidad y (B) sin rangos de proximidad, las columnas (1) y (2) exhiben las salidas de regresión para la estimación de la ecuación de precios hedónicos por Mínimos Cuadrados Ordinarios (OLS); en tanto, las columnas subsiguientes despliegan los hallazgos derivados de las estimaciones que toman en consideración la presencia de estructura espacial en el set de datos utilizado.

En particular, en (3) y (4), se exponen los resultados del modelo SEM por Máxima Verosimilitud (ML) -asumiendo distribución normal de la perturbación aleatoria-, en los que se verifica presencia de efectos directos y ausencia de efectos cruzados -i.e. spillovers-(LeSage y Pace, 2009). Y, por su parte, en (5) y (6) los correspondientes al modelo SARAR, que se comentarán en la próxima sección, dada la necesidad de profundizar en torno a los efectos directos e indirectos asociados. Este abordaje se conoce como "particular a general" (Hendry, 1995).

En relación a los dos primeros sets de atributos incluidos como variables explicativas, referidos a las características particulares del inmueble, y del edificio, los efectos hallados resultan estadísticamente significativos, y en línea con los obtenidos en la literatura especializada. A saber, en cuanto a los atributos particulares de la vivienda, se encontró una relación decreciente entre el precio por metro cuadrado y la superficie de la unidad habitacional. Resultado usual en este tipo de estimaciones, dado que en el caso de las
10. En particular, los estimadores obtenidos por MCO en presencia de un rezago espacial de la variable dependiente serán sesgados, en tanto que en el caso de autocorrelación espacial en los residuos de la regresión, los estimadores serán ineficientes (Anselin, 1988, p. 57 y ss.).

11. Bajo el cumplimiento de las condiciones: a) $|1-\rho W|>0$, b) $|I-\lambda W|>0$ y c) $\Omega_{\mathrm{ij}}>0$ para todo, siendo $\Omega$ la matriz de covarianzas del error (Anselin, 1988, p. 62; Elhorst, 2014, p.11). En nuestro caso se cumplen las tres condiciones. 
propiedades más grandes, no se cotizan simplemente multiplicando la cantidad de metros cuadrados por su precio, ya que en ciertos casos ello redundaría en un monto a partir del cual podrían adquirirse dos unidades más pequeñas y tener una renta.

Asimismo, se constata que la disposición al frente conlleva un efecto positivo y significativo, para un nivel de confianza del $99 \%$, que incrementa en términos promedio entre un 2,3\% y un 2,6\% el valor de la propiedad, según la especificación del modelo. En el mismo sentido operan las variables: lavadero y amoblado. En ambos casos, la incidencia positiva sobre el precio del inmueble de contar con lavadero y de venderse ya amoblado mueven al alza el valor del metro cuadrado ofertado, promediando tales movimientos $1,8 \%$ para las unidades con lavadero, y en torno a $10 \%$ para las viviendas amobladas.

De manera complementaria, puede verificarse que las variables dicotómicas asociadas a los barrios con valores extremos resultan significativas para todas las especificaciones, y que dan cuenta de diferencias de precio de magnitudes considerables: superiores al $35 \%$ para el caso de Puerto Madero, y hasta un 60\% menores para los barrios de Villa Lugano y Villa Soldati, y 27\% menor para la zona de Parque Patricios, siempre en comparación a los barrios comprendidos en el resto de la distribución.

Como se puede observar en el Cuadro 4, los resultados comentados presentan una pequeña variabilidad entre las especificaciones de los modelos y la técnica de estimación. No obstante, su significatividad estadística no se ve comprometida.

Cuadro 4. Resultados del modelo de precios hedónicos, según especificaciones. Fuente: elaboración propia.

\begin{tabular}{|c|c|c|c|c|c|c|}
\hline \multirow[t]{2}{*}{ Variables Explicativas } & \multicolumn{6}{|c|}{ Var. dependiente: Ipm2 (logaritmo del precio/m² en USD) } \\
\hline & OLS & SEM & SARAR & & & \\
\hline & $(\mathrm{A})$ & (B) & (A) & (B) & (A) & (B) \\
\hline \multirow[t]{2}{*}{ Superficie } & $-0,0002 * * *$ & $-0,0002 * * *$ & $-0,003 * * *$ & $-0,0003^{* * *}$ & $-0,0003^{* * *}$ & $-0,0003^{* * *}$ \\
\hline & $(0,0000)$ & $(0,0000)$ & $(0,0000)$ & $(0,0000)$ & $(0,0000)$ & $(0,0000)$ \\
\hline \multirow[t]{2}{*}{ Pisos } & $0,0106 * * *$ & $0,0102 * * *$ & $0,0104^{* * *}$ & $0,01 * * *$ & $0,0104^{* * *}$ & $0,0098 * * *$ \\
\hline & $(0,0005)$ & $(0,0005)$ & $(0,0005)$ & $(0,0005)$ & $(0,0005)$ & $(0,0005)$ \\
\hline \multirow[t]{2}{*}{ Pileta } & $0,0643^{* * * *}$ & $0,0641^{* * * *}$ & $0,069^{* * *}$ & $0,0696 * * *$ & $0,0692 * * *$ & $0,0706 * * *$ \\
\hline & $(0,0091)$ & $(0,0092)$ & $(0,009)$ & $(0,0091)$ & $(0,009)$ & $(0,0091)$ \\
\hline \multirow[t]{2}{*}{ Cochera } & $0,1057^{* * *}$ & $0,1085 * * *$ & $0,1115^{* * * *}$ & $0,1146^{* * *}$ & $0,1127^{* * *}$ & $0,1163^{* * *}$ \\
\hline & $(0,0067)$ & $(0,0067)$ & $(0,0066)$ & $(0,0067)$ & $(0,0066)$ & $(0,0066)$ \\
\hline \multirow[t]{2}{*}{ Gimnasio } & $0,1077 * * *$ & $0,1056 * * *$ & $0,1043^{* * *}$ & $0,1013^{* * *}$ & $0,1054 * * *$ & $0,1012^{* * * *}$ \\
\hline & $(0,0117)$ & $(0,0118)$ & $(0,0116)$ & $(0,0117)$ & $(0,0116)$ & $(0,0116)$ \\
\hline \multirow[t]{2}{*}{ Frente } & $0,0205^{* * *}$ & $0,0226 * * *$ & $0,0233^{* * *}$ & $0,0259^{* * * *}$ & $0,0243^{* * * *}$ & $0,0284^{* * *}$ \\
\hline & $(0,0063)$ & $(0,0064)$ & $(0,0063)$ & $(0,0063)$ & $(0,0062)$ & $(0,0063)$ \\
\hline \multirow[t]{2}{*}{ Vigilancia } & $0,0783 * * *$ & $0,0803 * * *$ & $0,0811 * * *$ & $0,0837 * * *$ & $0,0811 * * *$ & $0,084 * * *$ \\
\hline & $(0,0086)$ & $(0,0086)$ & $(0,0085)$ & $(0,0085)$ & $(0,0084)$ & $(0,0085)$ \\
\hline \multirow[t]{2}{*}{ Lavadero } & $0,0147^{* *}$ & $0,0143^{* *}$ & $0,0184 * * *$ & $0,0183^{* *}$ & $0,0203^{* * *}$ & $0,0197^{* * *}$ \\
\hline & $(0,0064)$ & $(0,0065)$ & $(0,0064)$ & $(0,0064)$ & $(0,0063)$ & $(0,0064)$ \\
\hline \multirow[t]{2}{*}{ Amoblado } & $0,1036 * * *$ & $0,1019 * * *$ & $0,1022 * * *$ & $0,0999^{* * * *}$ & $0,103 * * *$ & $0,1014 * * *$ \\
\hline & $(0,0172)$ & $(0,0173)$ & $(0,0171)$ & $(0,0173)$ & $(0,0171)$ & $(0,0172)$ \\
\hline \multirow[t]{2}{*}{ Subte (o-20omts) } & $-0,0473^{* * *}$ & & $-0,0491 * * *$ & & $-0,0515^{* * *}$ & \\
\hline & $(0,0084)$ & & $(0,0084)$ & & $(0,0084)$ & \\
\hline \multirow[t]{2}{*}{ Subte (200-30omts) } & $0,0158^{*}$ & & $-0,0165^{*}$ & & $-0,0158^{*}$ & \\
\hline & (o,009) & & $(0,0089)$ & & $(0,0088)$ & \\
\hline \multirow[t]{2}{*}{ Subte (300-6oomts) } & $0,0416 * * *$ & & $0,0362 * * *$ & & $0,0355^{* * *}$ & \\
\hline & $(0,0072)$ & & $(0,0072)$ & & $(0,0071)$ & \\
\hline \multirow[t]{2}{*}{ Subte (o-6oomts) } & & 0,0045 & & 0,0011 & & $-0,0007$ \\
\hline & & $(0,0063)$ & & $(0,0063)$ & & $(0,0063)$ \\
\hline
\end{tabular}




\begin{tabular}{|c|c|c|c|c|c|c|}
\hline \multirow[t]{2}{*}{ A estrenar } & $0,0607 * * *$ & $0,0621 * * *$ & $0,0494 * * *$ & $0,0511 * * *$ & $0,0426 * * *$ & $0,0439 * * *$ \\
\hline & $(0,0098)$ & $(0,0099)$ & $(0,0099)$ & $(0,01)$ & $(0,0099)$ & $(0,01)$ \\
\hline \multirow[t]{2}{*}{ Antigüedad 1-10 años } & $0,0776 * * *$ & $0,0817^{* * *}$ & $0,0748 * * *$ & $0,0779 * * *$ & $0,077^{* * * *}$ & $0,0801^{* * * *}$ \\
\hline & $(0,0092)$ & $(0,0092)$ & $(0,0091)$ & $(0,0092)$ & $(0,0091)$ & $(0,0091)$ \\
\hline \multirow{2}{*}{$\begin{array}{l}\text { Antigüedad 11-20 } \\
\text { años }\end{array}$} & $0,0775^{* * *}$ & $0,0798^{* * * *}$ & $0,0754^{* * * *}$ & $0,0768 * * *$ & $0,078^{* * *}$ & $0,079^{* * *}$ \\
\hline & $(0,012)$ & $(0,0121)$ & $(0,0118)$ & $(0,0119)$ & $(0,0118)$ & $(0,0119)$ \\
\hline \multirow{2}{*}{$\begin{array}{l}\text { Antigüedad 21-30 } \\
\text { años }\end{array}$} & 0,0025 & 0,0018 & 0,0024 & 0,0019 & 0,0043 & 0,0038 \\
\hline & $(0,0123)$ & $(0,0124)$ & $(0,0121)$ & $(0,0122)$ & $(0,012)$ & $(0,0121)$ \\
\hline \multirow[t]{2}{*}{ Antigüedad +41 años } & $-0,0049$ & $-0,0139^{*}$ & $-0,0032$ & $-0,0115$ & $-0,0006$ & $-0,0083$ \\
\hline & $(0,0081)$ & $(0,0081)$ & $(0,008)$ & $(0,008)$ & $(0,008)$ & $(0,008)$ \\
\hline \multirow[t]{2}{*}{ Barrio Puerto Madero } & $0,342 * * *$ & $0,3482 * * *$ & $0,353^{* * *}$ & $0,3696 * * *$ & $0,3321 * * *$ & $0,3589 * * *$ \\
\hline & $(0,0218)$ & $(0,022)$ & $(0,0219)$ & $(0,0222)$ & $(0,0219)$ & $(0,022)$ \\
\hline \multirow{2}{*}{$\begin{array}{l}\text { Barrio Lugano y } \\
\text { Soldati }\end{array}$} & $-6011 * * *$ & $-0,5976^{* * *}$ & $-0,6015^{* * *}$ & $-0,5982 * * *$ & $-0,5996 * * *$ & $-0,5968 * * *$ \\
\hline & $(0,071)$ & $(0,0716)$ & $(0,0699)$ & $(0,0704)$ & $(0,0695)$ & $(0,0699)$ \\
\hline \multirow{2}{*}{$\begin{array}{l}\text { Barrio Parque } \\
\text { Patricios }\end{array}$} & $-0,2768 * * *$ & $-0,2798^{* *}$ & $-0,2714 * * *$ & $-0,2736 * * *$ & $-0,2677^{* * *}$ & $-0,2691 * * *$ \\
\hline & $(0,0852)$ & $(0,0858)$ & $(0,0839)$ & $(0,0844)$ & $(0,0834)$ & $(0,0838)$ \\
\hline \multirow[t]{2}{*}{ Constante } & $7,6749 * * *$ & $7,6748 * * *$ & $7,6736 * * *$ & $7,6737 * * *$ & $7,6687 * * *$ & $7,6696 * * *$ \\
\hline & $(0,0099)$ & $(0,01)$ & $(0,0099)$ & $(0,01)$ & $(0,0099)$ & $(0,0099)$ \\
\hline \multirow[t]{2}{*}{ Rho } & & & $2,3878 * * *$ & $2,6998 * * *$ & $2,9835 * * *$ & $4,342^{* * *}$ \\
\hline & & & $(0,0858)$ & $(0,1109)$ & $(0,0873)$ & $(0,096)$ \\
\hline \multirow[t]{2}{*}{ Lambda } & & & & & $0,0881 * * *$ & $0,0767^{* * * *}$ \\
\hline & & & & & $(0,0091)$ & $(0,0057)$ \\
\hline \multirow[t]{2}{*}{ Sigma2 } & & & $0,0631 * * *$ & $0,064 * * *$ & $0,0624^{* * *}$ & $0,0631 * * *$ \\
\hline & & & $(0,0009)$ & $(0,001)$ & $(0,0009)$ & $(0,0009)$ \\
\hline Número de Obs. & 8098 & 8098 & 8098 & 8098 & 8098 & 8098 \\
\hline $\begin{array}{l}\text { Akaike Information } \\
\text { Criteria }\end{array}$ & 893,497 & 1012,759 & 677,614 & 782,635 & 685,048 & 592,031 \\
\hline
\end{tabular}

La antigüedad de las viviendas, en relación a la categoría base de propiedades de entre 31 y 40 años, presenta una no linealidad respecto a inmuebles más nuevos, pero no así para las más antiguas. Es decir, se puede observar que no existen diferencias estadísticamente significativas entre las viviendas de entre 31 y 40 años, y las más antiguas. Este es un resultado interesante, y que interpretamos como signo de una fuerte demanda latente en este mercado, atravesado en 2017 por un crecimiento exponencial de los créditos hipotecarios. El hecho de que en el precio de oferta no se refleje una disminución asociada a viviendas construidas en un momento previo a 1980 da cuenta de un convencimiento de que de todas maneras existirá un comprador dispuesto a pagar lo que sea necesario para residir en la Ciudad Autónoma de Buenos Aires. ${ }^{12}$

En contraste, si bien las unidades habitacionales más nuevas se asocian con precios más elevados, existe una diferencia entre las que son "A estrenar" y las que tienen cierta cantidad de años. En contraposición a lo que a priori esperábamos, el efecto es menos fuerte para las viviendas nuevas y sin uso (entre un $4,9 \%$ y un $5,1 \%$ ) y las que tienen una antigüedad menor a 30 años (entre un $7,5 \%$ y $7,8 \%$ ).

En lo respectivo a las características de los edificios, los resultados dan cuenta de una significativa influencia vinculada a los amenities. En particular, la disponibilidad de espacios de recreación, como pileta, presencia de vigilancia en el edificio y cochera propia, implican, en términos promedio un incremento en el precio por $\mathrm{m} 2$ de de la propiedad de entre 7\% y 11,5\%, según la combinación de amenities, respectivamente. 
Resulta interesante destacar que la valoración positiva de la vigilancia, además, tiene una representatividad heterogénea entre barrios, resultando un atributo mucho más ofrecido en barrios caros como Palermo o Belgrano.

La diferenciación entre especificaciones de los modelos tipo (A) y (B) nos permite ahondar en el tema de interés de la presente investigación. Un resultado que en principio parecería ser contra intuitivo, reflejado en las estimaciones OLS y SEM, es que el hecho de vivir en las cercanías de una estación de subte (hasta 600 metros) no resultaría en ningún tipo de diferencia traducida al valor del $\mathrm{m} 2$ de oferta para inmuebles en venta. Este resultado iría en dirección opuesta a la literatura especializada vinculada a señalar los beneficios derivados de la conectividad (Laakso, 1992). Sin embargo, un estudio más fino nos permite identificar un comportamiento no lineal, en el que se constata una penalidad sobre el valor del $\mathrm{m} 2$ de alrededor de -5\% derivado de una localización de extrema cercanía a la estación de subte (menor a 200 metros). Al respecto, en la literatura se plantea que el movimiento, el ruido y la contaminación de las inmediaciones a las estaciones pueden constituir motivos para encontrar allí precios menores a la zona de comparación (unidades a más de 600 metros de distancia), véase por ejemplo Gatzlaff y Smith (1993).

Siguiendo ese resultado, cabría pensar que en una ciudad como Buenos Aires, el transporte público se encuentra negativamente valorizado en las tasaciones de las propiedades, lo cual resultaría extraño, dado que en particular el subte es uno de los medios de transporte más utilizados. De allí que, en contraste, queremos dirigir nuestra atención al coeficiente estimado para las dos distancias categorizadas restantes (viviendas ubicadas entre 200 y 300 metros, y entre 300 y 600 metros de las estaciones). Puede observarse que se produce una reversión del efecto negativo antes señalado. De esta forma, la semi-elasticidad cambia de signo y representa un efecto parcial positivo de una magnitud de 3,6\%, para los modelos de regresión espacial. Entre los dos extremos de la estimación, la diferencia es de más de 8 puntos porcentuales, siendo ambas estimaciones significativas a $99 \%$ de confianza. El rango medio, en tanto, puede pensarse como un punto de inflexión ya que si bien la estimación arroja un resultado negativo, al 95\% de confianza este valor no es estadísticamente diferente de cero. Esta característica también se repite en las especificaciones que estiman el efecto promedio de todas las distancias, comentado anteriormente. La penalidad, pese a mostrar un valor negativo, no supera el test de significatividad individual. Si se compara con el resultado del modelo básico estimado por Mínimos Cuadrados Ordinarios, se puede ver que el coeficiente del efecto positivo del último tramo de distancia se encuentra sobreestimado en comparación.

\section{Efectos directos e indirectos para la estimación del Modelo SARAR}

Como se puede ver en el Cuadro 4, las estimaciones de los modelos que tienen en cuenta la dependencia espacial incluyen coeficientes adicionales que dan cuenta de la relevancia de los términos de rezago espacial en el error y en la variable dependiente. Estos son $\lambda$, para el Modelo SLM y $(\lambda, \rho)$ para el Modelo SARAR. En todas las estimaciones resultan significativos, aunque su significado es diferente. ${ }^{13}$

A diferencia de un modelo sin dependencia espacial estimado por OLS, en el Modelo SARAR la derivada parcial de $\mathrm{E}(\mathrm{Y})$ respecto del k-ésimo regresor tiene la particularidad de que si éste cambia para una observación $i$, ello redundará en un cambio no solamente en la variable dependiente para $i$, sino también para el resto de las observaciones. El primer cambio lleva el nombre de efecto directo, mientras que el segundo se denomina efecto indirecto. De allí que sobre cada observación pueda estimarse un efecto directo y otro indirecto. Siguiendo a LeSage y Pace (2009), lo que se reportará es únicamente el promedio simple de los efectos directos y de los efectos indirectos de cada regresor sobre la variable dependiente. ${ }^{14}$
14. De lo contrario, lo que se obtendría es un efecto directo, indirecto y total para cada observación. 
Formalmente, puede derivarse que los dos efectos, y su suma (Efecto Total) adquieren la siguiente especificación:

$$
\begin{array}{cc}
E T=\frac{\beta_{K}}{N} * \sum_{j=1}^{N} \sum_{i=1}^{N}\left[(I-\lambda W)^{-1}\right]_{i j}, & \forall i, j, \\
E D=\frac{\beta_{K}}{N} * \sum_{j=1}^{N} \sum_{i=1}^{N}\left[(I-\lambda W)^{-1}\right]_{i j}, & \forall i=j, \\
E I=\frac{\beta_{K}}{N} * \sum_{j=1}^{N} \sum_{i=1}^{N}\left[(I-\lambda W)^{-1}\right]_{i j}, & \forall i \neq j,
\end{array}
$$

El efecto directo considera los elementos de la diagonal principal de la matriz, en tanto que el efecto indirecto toma los elementos restantes. En nuestro caso, considerando que la cantidad de observaciones es 8098 , la suma del total de los elementos para los Modelos A y B es 8103,12 y 8102,45 , mientras que los elementos de la traza suman 8098,05 y 8098,03, respectivamente, con lo cual podemos intuir que los efectos indirectos serán poco significativos.

En efecto, como puede apreciarse en el Cuadro 5, los efectos contagio parecen ser insignificantes, $y$, por consiguiente, los impactos directos resultan prácticamente iguales a los efectos totales. En consecuencia, dado que el efecto multiplicador resulta irrelevante, lo que cabe concluir es que, según el criterio de Anselin y Florax (1995), habría fundamentos para elegir el Modelo SEM como aquel que mejor captura la dependencia

\begin{tabular}{|c|c|c|c|c|c|c|}
\hline \multicolumn{7}{|c|}{ Var. dependiente: Ipm2 (logaritmo del precio/m² en USD) } \\
\hline \multirow[t]{2}{*}{ Variables explicativas } & \multicolumn{3}{|c|}{ SARAR (A) } & \multicolumn{3}{|c|}{ SARAR (B) } \\
\hline & ET & ED & El & ET & ED & El \\
\hline Superficie & $-0,0002$ & $-0,0002$ & $\mathrm{o}$ & $-0,0003$ & $-0,0003$ & o \\
\hline Pisos & 0,0104 & 0,0104 & $\mathrm{O}$ & 0,0098 & 0,0098 & $\mathrm{o}$ \\
\hline Pileta & 0,0693 & 0,0692 & o & 0,0706 & 0,0706 & o \\
\hline Cochera & 0,1128 & 0,1127 & 0,0001 & 0,1164 & 0,1163 & 0,0001 \\
\hline Gimnasio & 0,1055 & 0,1054 & 0,0001 & 0,1013 & 0,1012 & 0,0001 \\
\hline Frente & 0,0244 & 0,0243 & 0 & 0,0284 & 0,0284 & o \\
\hline Vigilancia & 0,0811 & 0,0811 & 0,0001 & 0,084 & 0,084 & $\mathrm{o}$ \\
\hline Lavadero & 0,0203 & 0,0203 & o & 0,0198 & 0,0197 & o \\
\hline Amoblado & 0,103 & 0,103 & 0,0001 & 0,1015 & 0,1014 & 0,0001 \\
\hline Subte (o-20omts) & $-0,0515$ & $-0,0514$ & o & & & \\
\hline Subte (200-30omts) & $-0,0157$ & $-0,0157$ & 0 & & & \\
\hline Subte (300-6oomts) & 0,0355 & 0,0355 & o & & & \\
\hline Subte (o-6oomts) & & & & $-0,0007$ & $-0,0007$ & $\mathrm{o}$ \\
\hline A estrenar & 0,0426 & 0,0426 & 0 & 0,0439 & 0,0439 & o \\
\hline Antigüedad 1-10 años & 0,0771 & 0,077 & 0 & 0,0801 & 0,0801 & o \\
\hline Antigüedad 11-20 años & 0,078 & 0,078 & $\mathrm{O}$ & 0,0791 & 0,079 & $\mathrm{o}$ \\
\hline Antigüedad 21-30 años & 0,0043 & 0,0043 & o & 0,0038 & 0,0038 & o \\
\hline Antigüedad +41 años & $-0,0006$ & $-0,0006$ & o & $-0,0083$ & $-0,0083$ & o \\
\hline Barrio Puerto Madero & 0,3323 & 0,3321 & 0,0002 & 0,3591 & 0,3589 & 0,0002 \\
\hline Barrio Lugano y Soldati & $-0,6$ & $-0,5996$ & $-0,0004$ & $-0,5971$ & $-0,5968$ & $-0,0003$ \\
\hline Barrio Parque Patricios & $-0,2678$ & $-0,2676$ & $-0,0002$ & $-0,2693$ & $-0,2691$ & $-0,0001$ \\
\hline Constante & 7,6735 & 7,6687 & 0,0048 & 7,6738 & 7,6697 & 0,0042 \\
\hline
\end{tabular}
espacial, aún cuando se rechazare la hipótesis nula de ambos tests LM.

Cuadro 5. Discriminación entre efectos totales, directos e indirectos. Fuente: Elaboración propia.

Otro indicio de que ésta sería la decisión correcta puede deducirse de la observación del Criterio de Información de Akaike (1973) (AIC). En el Cuadro 4 se muestra que 
para la especificación (A), que es la que resulta relevante a los propósitos de esta investigación dado que se analiza la relevancia de las no linealidades encontradas, el AIC del Modelo SEM es de 677,6 contra 685,04 del Modelo SARAR, reforzando el primer criterio considerado. Si bien la diferencia es pequeña, refuerza la evidencia a favor del Modelo de Error Espacial.

\section{Reflexiones finales}

Retomando las discusiones referidas a las plusvalías urbanas, y considerando la categorización planteada entre valorización inmobiliaria como resultado de esfuerzo individual (propio o de terceros) y como resultado de acción gubernamental, en el presente estudio se evalúa la incidencia de la infraestructura de transporte subterráneo sobre los valores de inmuebles ofertados para su venta, para la Ciudad Autónoma de Buenos Aires.

Las plusvalías urbanas refieren a valorizaciones inmobiliarias generadas socialmente. De allí que la literatura especializada se haya concentrado en analizar las formas e instrumentos social y políticamente viables para su captación. Dichas herramientas pueden imbuir a la política fiscal de una impronta relevante en términos distributivos, en tanto su objetivo ulterior radica en recuperar para las arcas públicas, al menos parcialmente, las ganancias derivadas del aumento de precios de los inmuebles ligado a acciones del Estado, no vinculadas con el propietario individual.

No obstante, una instancia inaugural para la planificación de las estrategias de captación de plusvalías radica en la capacidad de, por un lado mensurar correctamente las valorizaciones, y por otro, identificar sobre qué propiedades se produce el efecto riqueza.

En ese sentido, se procedió inicialmente a analizar en términos descriptivos las heterogeneidades existentes entre los precios de inmuebles en las proximidades de las seis líneas que conforman la red de subterráneos. El cálculo de los precios promedio por metro cuadrado para un radio de 600 metros de cercanía a cada una de las estaciones de la red de subterráneos arroja así un primer indicio en relación a la presencia de una significativa dispersión de guarismos entre líneas. Sin embargo, lo propio también ocurre entre las bocas que componen cada ramificación de la red: en términos promedio, el valor del metro cuadrado para inmuebles -entre 0 a 20 años de antigüedad- en venta cercanos a la Línea $\mathrm{D}$ resulta un $60,1 \%$ superior al de inmuebles contiguos a la Línea $\mathrm{E}$, un $46,1 \%$ por encima de los de la línea $\mathrm{A}$, un $45,9 \%$ respecto a los de la línea $\mathrm{H}$, un $34,2 \%$ en relación a los de la línea B, y un $17,7 \%$ por encima de los de la línea C.

Complementando la evidencia precedente, se avanzó en la medición y análisis del efecto que la proximidad a la red de transporte subterráneo detenta sobre las propiedades en venta en la Ciudad Autónoma de Buenos Aires, considerando la presencia de estructuras espaciales en la muestra utilizada -de 8098 inmuebles ofertados entre Septiembre y Diciembre de 2017- y distintos tramos de distancia a las bocas de subte a) muy próximo al subte, inmuebles en el rango 0-200 metros del subte; b) próximo al subte, inmuebles en el rango 200-300 metros del subte; c) cercano al subte, inmuebles en el rango 300-600 metros del subte; d) en el potencial radio de influencia del subte, inmuebles en el rango 0-600 metros del subte-; e) el resto de la ciudad, con miras a evaluar la presencia de no linealidades.

Las estimaciones efectuadas a través del método de precios hedónicos permiten explicar la relevancia de distintos atributos -i.e. características individuales, del edificio y de localización/conectividad- sobre el valor del precio por metro cuadrado de las 
propiedades. Sin embargo, en caso de que la base de datos presente dependencia espacial, el modelo de precios hedónicos no puede estimarse mediante Mínimos Cuadrados Ordinarios. Una de las contribuciones de la presente investigación consiste en efectuar una primera estimación a través del uso de econometría espacial de las especificidades del Mercado Inmobiliario de venta para la Ciudad Autónoma de Buenos Aires, presentando un estudio en detalle de la identificación de plusvalías urbanas asociadas al transporte público subterráneo. En consecuencia, dado que se halló la presencia de una estructura espacial inherente al set de datos, es decir que los valores de las propiedades se agrupan por proximidad, y no de forma aleatoria, las estimaciones que han de tomarse en consideración son las correspondientes al Modelo de Error Espacial (SEM) y al Modelo autorregresivo con residuos autorregresivos (SARAR); aunque de acuerdo a los criterios de selección analizados, el SEM emerge como el preferido.

En relación a los atributos particulares y del edificio, los resultados obtenidos se encuentran en línea con los de investigaciones previas: a) la disponibilidad de espacios de recreación, como pileta, presencia de vigilancia en el edificio y cochera propia, implican, en términos promedio un incremento en el precio por $\mathrm{m} 2$ de oferta de la propiedad de entre 7\% y 11,5\%, según la combinación de amenities; b) en relación a la antigüedad, tomando como categoría base las unidades de entre 31 y 40 años, se verifica una no linealidad en relación a las unidades más nuevas, de manera que si la vivienda es a estrenar, en promedio, el valor del $\mathrm{m}^{2}$ se eleva entre un $4,9 \%$ y un $5,1 \%$, mientras que para aquellas con menos de 30 años de antigüedad, ese impacto asciende a 7,5\% y 7,8\%; c) el efecto de residir en una determinada zona de la ciudad -que miden las variables dicotómicas vinculadas a los barrios en los que se concentran los guarismos extremos de la variable a explicar- resulta un elemento clave para la definición del valor del $\mathrm{m} 2$, dando cuenta de diferenciales positivos del orden del $35 \%$ para inmuebles localizados en Puerto Madero, y negativos para Villa Lugano y Soldati, del orden del -60\% y -27\%, respectivamente.

Por su parte, en relación a las variables de interés, encontramos que, al considerarse un buffer -radio de influencia- de 600 metros, el efecto no resulta estadísticamente significativo. No obstante, al incluirse distintos tramos de proximidad se constata que este resultado esconde una dinámica heterogénea en función de distintas distancias: si bien la presencia de una boca de subte en las inmediaciones de las propiedades ( 0 a 200 metros) detenta una incidencia negativa del 5\% -probablemente explicado por el efecto congestión-, dicho resultado se revierte cuando se trata de inmuebles que, aunque contiguos, no se hallan lo suficientemente cerca del subterráneo (entre 300 y 600 metros), para los cuales el impacto de la localización incrementa el valor del m2 en un 3,6\%. Es decir, se verifica una no linealidad que, entre los extremos de distancia estimada, arroja una diferencia de más de 8 puntos porcentuales sobre el valor del metro cuadrado. Estos resultados, además, podrían constituir un elemento clave para discutir el efecto riqueza positivo resultante de la acción del Estado, y en simultáneo poner de manifiesto la presencia de un efecto en sentido contrario, que implica que para propiedades ubicadas en un rango de extrema proximidad a las bocas de subte se constata una reducción en el valor del metro cuadrado de oferta. Esta incidencia negativa derivada de la intervención estatal resulta un aspecto que escasamente explorado, tanto en la literatura como en las estrategias de captación (en este caso, también compensación) de plusvalías urbanas.

De hecho, con miras a contribuir a las discusiones en torno a la valorización inmobiliaria generada a partir de intervenciones gubernamentales -como es el caso de la provisión de infraestructura de transporte-, estos resultados representan un insumo fundamental para el adecuado diseño de una política pública específica para la Ciudad Autónoma de Buenos Aires. Para finalizar, los resultados encontrados constituyen un elemento adicional para explicar las heterogeneidades referentes al precio de la vivienda 
en los diferentes barrios de la CABA. Incluso al interior de un mismo barrio, como fue explicado aquí, existe una dispersión de precios, con un diferencial promedio positivo ubicado en el rango de 300 a 600 metros de las estaciones de subte. A los fines de la recuperación de las plusvalías urbanas, por lo tanto, se deberá tener en cuenta para aplicar una política tributaria equitativa, diferenciada en términos de localización. Consecutivamente, configura el camino para una agenda de investigación direccionada hacia la identificación de: a) diferencias en el efecto riqueza encontrado respecto a distintas líneas de subterráneo; y b) especificidades equivalentes en otras ciudades, tanto en lo que respecta a la política de transporte, como así también al resto de políticas urbanas.

Agradecimientos: Los autores agradecen los valiosos y fructíferos comentarios, sugerencias y discusiones de Gustavo Ludmer, Pablo Wahren, Damián Rosanovich, Martín Ferroni, Verónica Daverio y Lisandro Mondino en versiones preliminares de esta investigación. Cualquier error u omisión remanente es exclusiva responsabilidad de los autores. Las traducciones son nuestras. 


\section{Q Bibliografía}

"Akaike, H. (1973). Information Theory and an Extension of the Maximum Likelihood Principle, Second International Symposium on Information Theory, B. Petrov y F. Csaki (edits.), Budapest.

"Alonso, W. (1960). A theory of the urban land market. Papers in Regional Science 6 (1), 149-157.

»Anselin, L. (1988). Spatial econometrics: methods and models. Kluwer Academic Publishers: Holanda.

»Anselin, L. (2013). Spatial econometrics: methods and models. Berlin: Springer Science \& Business Media.

»Anselin, L., Bera, A., Florax, R. y Yoon, M. (1996). Simple diagnostic tests for spatial dependence. Regional science and urban economics 26 (1), 77-104.

"Anselin, L. y Bera, A. (1998).Spatial dependence in linear regression models with an application to spatial econometrics. En: Ullah A, G. (eds). Handbook of applied economics statistics. Berlin: Springer.

"Anselin, L. y Florax, R. (1995). Small sample properties of tests for spatial dependence in regression models: Some further results. En: New directions in spatial econometrics. Springer: Berlin.

»Anselin, L. y Rey, S. (1991). Properties of tests for spatial dependence in linear regression models. Geographical analysis 23 (2), 112-131.

» Baer, L (2012). Mercados de suelo y producción de vivienda en Buenos Aires y su área metropolitana: tendencias recientes de desarrollo urbano y acceso a la ciudad. Revista Iberoamericana de Urbanismo 8, 43-58.

»Baer, L. y Kauw, M. (2016). Mercado inmobiliario y acceso a la vivienda formal en la Ciudad de Buenos Aires, y su contexto metropolitano, entre 2003 y 2013. Revista EURE 42 (126), 5-25.

" Bajic, V. (1983). The effects of a new subway line on housing prices in metropolitan Toronto. Urban studies 20 (2), 147-158.

»Bowes, D. y lhlanfeldt, K. (2001). Identifying the impacts of rail transit stations on residential property values. Journal of Urban Economics 50 (1), 1-25.

» Burkhardt, R. (1976). Summary of Research: Joint Development Study. New York: Administration and Managerial Research Association.

"Castillo, M. (2011). Efecto de la cercanía a las estaciones de Subte y valor de la propiedad residencial en Buenos Aires. Café de las Ciudades 10 (101), disponible en: http://www.cafedelasciudades.com.ar/economia_101.htm.

»Cervero, R. y Landis, J. (1995). Development impacts of urban transport: a US perspective. En: D. Banister (ed.). Transport and urban development, E \& FN Spon: Londres, 136-156.

»Chaney, T., Sraer, D. y Thesmar, D. (2012). The collateral channel: How real estate shocks affect corporate investment. American Economic Review 102 (6), 2381-2409.

»Clichevsky, N. (2001). La captación de plusvalías urbanas en América Latina: ¿futuro instrumento de equidad social?. En: Smolka y Furtado (eds). 
Recuperación de plusvalías en América Latina. Santiago de Chile: Eure libros Lincoln Institute of Land Policy, 1-35.

"Clichevsky, N. (2004). Algunos instrumentos para la captación de plusvalías. Ciudad y territorio Estudios Territoriales 36 (140), 459-460.

»Conte Grand, M. (2011). Una primera aproximación a la valuación hedónica de la contaminación en Buenos Aires. Serie Documentos de Trabajo, Universidad del CEMA: Área: economía 207, 1-21.

»Cuenya, B. y Corral, M. (2011). Empresarialismo, economía del suelo y grandes proyectos urbanos: el modelo de Puerto Madero en Buenos Aires. Eure 37 (111), $25-45$.

"Damm, D., Lerman, S., Lerner-Lam, E. y Young, J. (1980). Response of urban real estate values in anticipation of the Washington Metro. Journal of Transport Economics and Policy 14 (3), 315-336.

» Debrezion, G., Pels, E., Rietveld, P. (2007). The impact of railway stations on residential and commercial property value: a meta-analysis. The Journal of Real Estate Finance and Economics 35 (2), 161-180.

»De Gallo, J. (2014). Cross-Section Spatial Regression Models. En Fischer, M. y Nijkamp, P. (ed.): Handbook of regional science. Heidelberg: Springer, 1511-1532.

"Dornbush, D. (1975). BART-Induced Changes in Property Values and Rents, in Land Use and Urban Development Projects: Phase I, BART Impact Study. U.S. Department of Housing and Urban Development and U.S. Department of Transportation.

» Downing, P. (1973). Factors affecting commercial land values: an empirical study of Milwaukee, Wisconsin. Land Economics 49 (1), 44-56.

» Drukker, D., Peng, H., Prucha, I. y Raciborski, R. (2013). Creating and managing spatialweighting matrices with the spmat command. Stata Journal 13 (2), 242-286.

»Dunn, E. (1954). The Location of Agricultural Production. University of Florida Press, Gainsville, Florida.

"Elhorst, P. (2014). Linear spatial dependence models for cross-section data. Spatial Econometrics. Springer, Berlin, Heidelberg, 5-36.

» Fernandes, E., Maldonado, M. (2009). Law and Land Policy in Latin America Shifting Paradigms and Possibilities for Action. Lincoln Institute of Land Policy Land Lines - July, 1-6.

» Fischer, M., Nijkamp, P. (eds). (2014). Handbook of regional science. Heidelberg: Springer.

»Freeman, M. (1979). Hedonic Prices, Property Values and Measuring Environmental Benefits: A Survey of the Issues. The Scandinavian Journal of Economics 81 (2), 154-173.

" Fujita, M., Krugman, P., Venables, A. (1999). Economía Espacial: Las Ciudades, Las Regiones y el Comercio Internacional. Barcelona: Ariel Economía.

"Fuks, A., Senderovsky, D. y Suez, M. (2012). Los determinantes del precio de la propiedad en la Ciudad de Buenos Aires, Tesis de Maestría. Universidad Torcuato Di Tella.

»Furtado, F. (1999). Recuperação de mais-valias fundiárias urbanas na América Latina: debilidade na implementação, ambigüidades na interpretação. Tesis de doctorado. São Paulo: FAUUSP. 
» Gatzlaff, D. y Smith, M. (1993). The Impact of the Miami Metrorail on the Value of Residences near Station Locations. Land Economics 69 (1), 54-66.

» Getis, A. (2009). Spatial weights matrices. Geographical Analysis 41 (4), 404-410.

"Gobierno de la Ciudad de Buenos Aires. (2006). Diferencias y nuevos espacios territoriales. En Anuario 2006. Disponible en: http://www.estadisticaciudad. gob.ar/eyc/publicaciones/anuario_2006/tomo1/10.pdf

"Gomez Mera, M. (1998). El valor social de las plazas. Tesis de Maestría. Universidad de San Andrés.

»Goodman, A. (1978). Hedonic prices, price indices and housing markets. Journal of urban economics 5 (4), 471-484.

» Haig, R.(1926). Toward an understanding of the Metropolis: II. The assignment of activities to areas in urban regions. The Quarterly Journal of Economics 40 (3), 402-434.

» Hendry, D. (1995). Dynamic econometrics. Oxford University Press, Oxford.

» Herrera, M. (2015). Econometría especial usando Stata. Breve guía aplicada para datos de corte transversal. Documentos de Trabajo del IELDE 13, 1-55.

»Isard, Walter. (1956). Location and Space-Economy. John Wiley \& Sons, New York.

» Jaramillo, S. (1994). Los fundamentos económicos de la "participación en plusvalías". Boston: Lincoln Institute of Land Policy.

» Jaramillo, S. (2003). Los fundamentos económicos de la participación en plusvalías. Bogotá: CEDE-Universidad de los Andes, Lincoln Institute of Land Policy.

" Jaramillo, S. (2006). Recuperación de plusvalías en Colombia. Reflexiones sobre su desarrollo histórico. Cadernos IPPUR 9.

» Jaramillo, S. (2008[1977]). Hacia una teoría de la renta del suelo urbano. Colombia: EdicionesUniandes-Universidad de los Andes.

»Laakso, S. (1992). Public transport investment and residential property values in Helsinki. Scandinavian Housing and Planning Research 9 (4), 217-229.

"Lesage, J. y Pace, K. (2009). Introduction to Spatial Econometrics (Statistics, textbooks and monographs). Boca Raton: CRC Press.

" Linneman, P. y Wachter, S. (1989). The impacts of borrowing constraints on homeownership. Real Estate Economics 17 (4), 389-402.

»Loaiza Rincón, L. y Morales, C. (2002). El trolebús como una política pública. Fermentum. Revista Venezolana de Sociología y Antropología 12 (34), 271-284.

» Mattos, L. (2006). Nova Ordem Jurídico-Urbanística: Função social da propriedade na prática dos tribunais. Rio de Janeiro: Lumen Juris.

" Medda, F. y Modelewska, M. (2011). Land value capture as a funding source for urban investment: The Warsaw metro System. Ernst \& Young Report - Better Goverment Program 2009-2010.

»Meloni, O. y Ruiz Nuñez, F. (1997). Determinantes de los Precios de Mercado de los Terrenos en San Miguel de Tucumán. A.A.E.P.: Anales: XXXIII Reunión Anual, Mendoza.

» Mera, K. (2016). Asia's financial crisis and the role of real estate. London: Routledge.

»Morales Schechinger, C. (2005). Conceptos avanzados de suelo urbano. Mercado del Suelo Urbano en Ciudades Latinoamericanas y Financiamiento de 
Ciudades Latinoamericanas con Suelo Urbano, Lincoln Institute of Land Policy.

" Moran, P. (1950). A test for the serial independence of residuals. Biometrika 37 (1/2), 178-181.

» Munévar, C. y Hernández, N. (2017). La naturaleza jurídica de la plusvalía urbana. Un análisis desde los derechos colectivos y la función social de la propiedad. Opción 33 (82), 280-297.

»Orduna, M. et al. (2016). Impacto del "metrobús" sobre el corredor Juan B. Justo de Buenos Aires. Observatorio de Sostenibilidad Urbana. Disponible en http:// repositorio.ub.edu.ar/handle/123456789/8328.

»Prévôt Shapira, M. (2002). Buenos Aires en los años 90: metropolización y desigualdades. Revista EURE 28 (85), 31-50.

" Ricardo, D. (1817). Principios de economía política y tributación. México: Fondo de Cultura Económica, 1959.

» Rice Center, (1987). Assessment of Changes in Property Values in Transit Areas. Joint Center for Urban Mobility Research-Prepared for the Urban Mass Transit Administration.

» Rodriguez, M. y Di Virgilio, M. (2014). Políticas de tierra y vivienda y déficit habitacional en el Área Metropolitana de Buenos Aires. Estudos de Sociología 1 (15), 57-83.

"Roncaglia, A. (2006). La riqueza de las ideas: una historia del pensamiento. Zaragoza: Prensas Universitarias de Zaragoza.

» Rosen, S. (1974). Hedonic prices and implicit markets: product differentiation in pure competition. Journal of Political Economy 82 (1), 34-55.

"Sassen, S. (1991). The Global City: New York, London, Tokyo. Princeton: Princeton University Press.

»Sheppard, S. (1999). Hedonic analysis of housing markets. Handbook of regional and urban economics 3, 1595-1635.

"Smith, A. (2007 [1776]). La riqueza de las naciones. Madrid: Alianza.

"Smith, J. y Gihring, T. (2006). Financing transit systems through value capture. American Journal of Economics and Sociology 65 (3), 751-786.

»Spengler, E. (1930). Land values in New York in relation to transit facilities. New York: Columbia University Press.

»Szajnberg, D. (2006). El proceso de densificación del tejido residencial en Buenos Aires desde los años noventa (Tesis de Maestría en Planificación Urbana y Regional), Facultad de Arquitectura, Diseño y Urbanismo, Universidad de Buenos Aires.

» Teubal, M. (2012). La renta de la tierra en la economía política clásica: David Ricardo. Revista Nera 8, 122-132.

» Topalov, C. y Enrique, F. (1984). Ganancias y rentas urbanas. Elementos teóricos. Madrid: Siglo XXI.

"Von Thünen, J. (1826). Isolated State. Londres: Pergamon press, 1966.

»Wagner, R. (2009). La administración de una ciudad más justa e inclusiva. Nuevos instrumentos de planificación y desarrollo urbano. RED MUNI $X$ Seminario, Nuevo rol del Estado, nuevo rol de los Municipios. 13 y 14 de agosto de 2009. UNLM. 


\section{Sergio Andrés Rosanovich / s.rosanovich@gmail.com}

Licenciado en Economía (UBA) y Magister en Desarrollo Económico (UNSAM). Ha publicado artículos vinculados a la distribución del ingreso, la inflación y el tipo de cambio. Actualmente desarrolla su investigación de doctorado en el Instituto de Altos Estudios Sociales (IDAES-UNSAM) sobre suelo urbano, mercados de venta y alquiler incorporando elementos de programación y métodos cuantitativos. Es ayudante de primera en Estadística II (UBA).

\section{Ana Paula Di Giovambattista / anapauladigio@gmail.com}

Licenciada en Economía (UNLP). Ha publicado trabajos sobre distribución del ingreso, desarrollo económico y dinámica de valorización del suelo en Argentina. En la actualidad, lleva a cabo tareas de investigación en el Centro de Innovación de los Trabajadores (CITRA) - (UMET - CONICET) en torno al acceso a la vivienda y hábitat y mercado inmobiliario. Asimismo, se desempeña como docente de Economía y Macroeconomía Superior (UNM). 